\title{
SYNTHESIS AND ANTINEOPLASTIC ACTIVITY OF CERTAIN TRIAZENE AND TRIAZENO-ACRIDINE COMBILEXIN DERIVATIVES
}

Samir M. El-Moghazy Aly ${ }^{1}$, Hanan H. Georgey ${ }^{1}$, Mohammed A. Mohammed $^{1}$, Nagwa M. Abdel Gawad ${ }^{1}$ and Noha H. Amin ${ }^{2}$

Department of Pharmaceutical Chemistry, Faculty of Pharmacy, ${ }^{1}$ Cairo University and ${ }^{2}$ Bani-Sueif University, Egypt

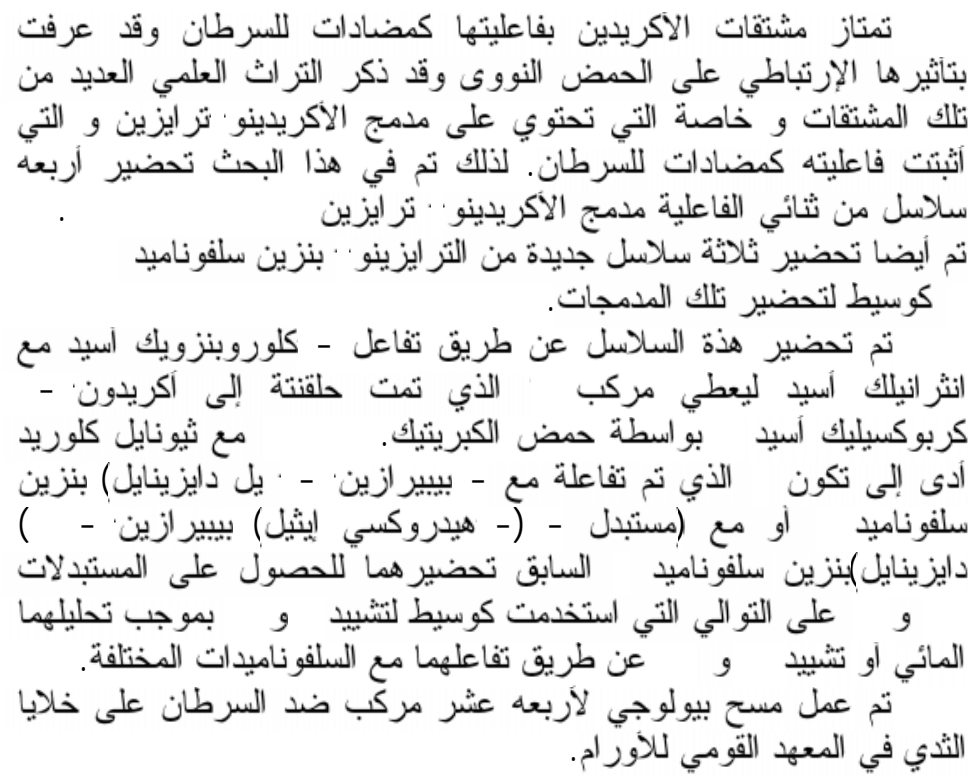

Four series of bifunctional ligands have been synthesized as DNA-binding combilexins. These novel agents contain a triazenobenzene sulfonamide linker moiety that is attached to an intercalating acridine or acridone chromophore by a functionalized amide or ester residue. In order to obtain these combilexins three series of the anticipated antitumor triazeno-benzene sulfonamide were synthesized. The synthesis and bioscreening of the new antineoplastic compounds are depending on the structural correlation with several reported antineoplastic acridines.

2-Chlorobenzoic acid was reacted with anthranilic acid to give $\mathrm{N}$-(2-carboxyphenyl) anthranilic acid which upon cyclodehydration 
with sulfuric acid afforded 9-oxo-9,10-dihydroacridine-4carboxylic acid, (acridone-4-carboxylic acid) 8. This latter intermediate has been converted to 9-chloroacridine carbonyl chloride 9 using thionyl chloride. Selective substitution of 9 with derivatives of 4-(piperazine-1-yldiazenyl) benzenesulfonamides $\mathbf{4 a -}$ $\boldsymbol{e}$ or derivatives of 4-(2-hydroxyethyl)piperazine-1-yl)diazenyl) benzenesulfonamides 5a-e to yield their 9-chloroacridine-4carboxamides 10a-e and 9-chloroacridine-4-carboxylic acid esters 13a-e respectively. Those intermediates have been reacted either with different sulfonamides to give derivatives of 4-\{4-[4-(4 sulfamoylphenyldiazenyl)piperazine-1-carbonyl]-9-ylamino\}benzenesulfonamides 11a-h and derivatives of 2-[(4-(4-sulfamoylphenyl)diazenyl)piperazine-1-yl]ethyl 9-(4-sulfamoylphenylamino)-9,10-dihydroacridine-4-carboxylates $14 a-i$ respectively or subjected to mild acid hydrolysis to yield derivatives of 4-\{4-[(9oxo-9,10-dihydroacridine-4-carbonyl)piperazine-1-yl]diazenyl $\}$ -

benzenesulfonamide 12a-e and derivatives of 2-\{4-[(4-sulfamoylphenyl)diazenyl]piperazine-1-yl\}ethyl-9-oxo-9,10-dihydroacridine4-carboxylate 15a-e respectively.

Besides, the synthesis of derivatives of 4-(piperazine-1yldiazenyl) benzenesulfonamides 4a-e and derivatives of 4-(2hydroxyethyl)piperazine-1-yl)diazenyl) benzenesulfonamides 5a-e has been achieved via diazotization of various substituted benzene sulfonamides with sodium nitrite and hydrochloric acid followed by various amines coupling to yield the target triazeno-benzene sulfonamides.

Fourteen new compounds were selected for screening their antitumor activity against breast cell line in National Cancer Institute. Six of them were found to be active as antitumor agents, while two were found to be mild active.

\section{INTRODUCTION}

Many publications describe the biological importance of acridine derivatives as antitumor agents ${ }^{1 \& 2}$, which are related to their ability to intercalate $\mathrm{DNA}^{3}$. The binding energy is obtained by insertion a flat aromatic portion of the nucleus between the base pairs of DNA double helix ${ }^{4 \& 5}$, that causes DNA break and chromosome damage ${ }^{6}$. The DNA binding propensities and topoisomerase II-inhibitory activities of acridines have been exploited in the development of clinically-active antitumor agents ${ }^{7}$.

9-Anilinoacridine derivatives have been extensively studied and one congener, namely m-AMAS I has become useful in clinical application for treatment of leukemia and 
lymphoma $^{8 \& 9}$. Furthermore, the introduction of 4-carboxamide group such as compound II have led to increase in the activity ${ }^{10 \& 11}$.<smiles>COc1cc(N(C)C(C)(C)C)ccc1Nc1c2ccccc2nc2ccccc12</smiles>

I<smiles>CNC(=O)c1cccc2c(Nc3ccc(N(C)C(C)=O)cc3OC)c3cccc(C)c3nc12</smiles>

II

On the other hand, a class of alkylating agents represented by triazene compounds ${ }^{12-14}$, are able to alkylate DNA molecule, acting mainly on the N-7 or O-6 atoms of guanine ${ }^{15 \& 16}$ as diazenopiperazine derivatives III $^{17 \& 18}$. Additionally, the formation of acridine-triazene combilexins IV and $\mathbf{V}^{19 \& 20}$ showed potential antitumor activity.

Based on the aforementioned facts, it was intended to investigate the synthesis of the new triazene derivatives 3-5 and the acridinetriazene combilexin derivatives $\mathbf{1 1}$ and $\mathbf{1 4}$ have been synthesized<smiles>CN1CCN(N=Nc2ccc(N)cc2)CC1</smiles>

$\mathrm{R}=\mathrm{CH}_{3}, \mathrm{CH}_{2} \mathrm{CH}_{2} 1, \mathrm{COOC}_{2} \mathrm{H}_{5}$

III

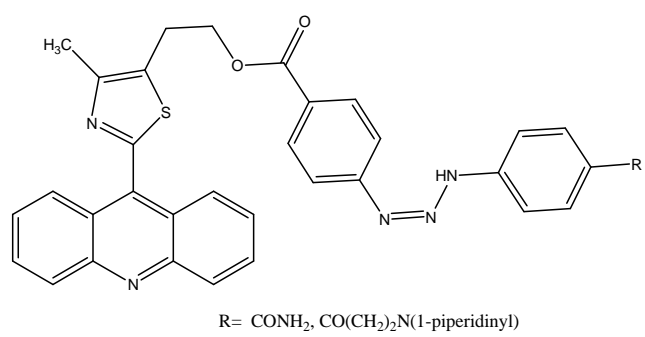

IV

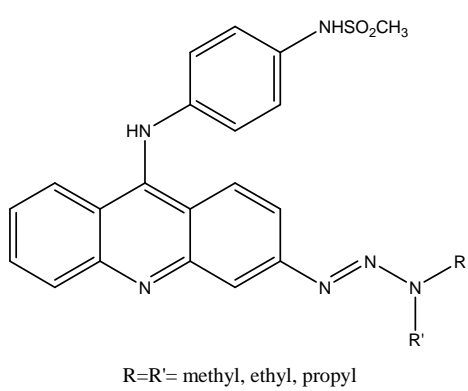

V

containing a triazene moiety, which itself possesses a cytotoxic activity, linked by an amide or ester linkage in the 4-position to the active 9substituted anilinoacridine analog. Such hybridization between the self active moieties was expected to 
enhance the observed overall antineoplastic profile.

Nevertheless, the presence of a carboxamide side chain, which attached to a terminal ring peri to an electron withdrawing atom in a linear tricyclic chromophore, represents an interesting class of DNA intercalator $^{21}$. Among this, amide derivative VI which has shown significant antitumor activity and a subpanel disease selectivity against prostate cancer $^{22}$. As a result, compounds 12 have been prepared in which the 4-carboxamido moiety of VI is replaced by the piperazinocarbonyl function.

Finally, acridone-4-acetic acid VII showed significant antitumor activity $^{23}$. Consequently, the effect of methyl removal together with the formation of prodrug containing the anticipated antitumor triazenopiperazine side chain have been studied through the synthesis of compound 15.<smiles>Nc1ccc(NC(=O)C2=CC=CC3C(=O)c4ccccc4NC23)cc1</smiles>

$\mathrm{R}=$ different heterocycles<smiles>O=C(O)CC1=CC=CC2C(=O)c3ccccc3NC12</smiles>

\section{CHEMISTRY}

The target compounds $\mathbf{3}, \mathbf{4}, \mathbf{5}, \mathbf{1 1}$, 12, 14, and 15 were synthesized as outlined in schemes 1,2 and 3 . The synthesis of compounds $\mathbf{3}$ and $\mathbf{5}$ has been achieved via diazotization of various substituted benzene sulfonamides followed by coupling with ethoxycarbonyl piperazine or with hydroxyethylpiperazine in a neutral medium adjusted with sodium acetate $^{18}$. The structure of the $\mathbf{3}$ and $\mathbf{5}$ was established through spectroscopic (IR, ${ }^{1} \mathrm{H}-\mathrm{NMR}$, Mass) as well as elemental analyses data. IR spectra of 3a-e and 5a-e revealed the presence of $\mathrm{NH}$ band $(=3279-3448), \quad \mathrm{SO}_{2}$ groups (= 1133-1159 and 1308-1356) in addition to the triazene band (=1422-1439). ${ }^{1} \mathrm{H}-\mathrm{NMR}$ revealed the presence of aromatic protons $(=7.29$ 7.93), the characteristic two triplet piperazine protons at $(=2.49-3.73$ and 3.72-3.39) beside the presence of the triplet and quartet ethyl protons of $\mathbf{3}$ $(=1.23$ and 4.07-4.09) or the presence of the triplet ethylene protons of $5(=2.57-2.72$ and 2.883.35). Mass spectra of compounds 3d and $\mathbf{5 a}$ showed the molecular ion peaks of the compounds $\left(=383\left(\mathrm{M}^{+}\right)\right.$ and $313\left(\mathrm{M}^{+}\right)$respectively).

Furthermore, alkaline hydrolysis of compound $\mathbf{3}$ followed by decarboxylation led to the monosubstituted piperazine derivatives 4 . IR spectra of compounds $4 \mathbf{a}-\mathbf{e}$ revealed the disappearance of the ester carbonyl band (=1683-1652). ${ }^{1} \mathrm{H}-\mathrm{NMR}$ revealed the disappearance of the characteristic ethyl protons. 
Mass spectra of compounds $\mathbf{4 a}$ and 4d showed the molecular ion peaks of the compounds $\left(=269\left(\mathrm{M}^{+}\right)\right.$and 313 $(\mathrm{M}+2)$ respectively).

9-Chloroacridine-4-carbonyl

chloride 9 was reacted with the triazenosulfonamide derivatives 4a-e or 5a-e in N,N-dimethylformamide in presence of triethylamine to yield 10a-e or 13a-e that was directly added to acidified solution of the appropriate sulfonamides in $\mathrm{N}, \mathrm{N}$ dimethylformamide to obtain 11a-h or 14a-j respectively. IR spectra showed the appearance of $\mathrm{NH}$ band $(=3427-3100)$, carbonyl band (= 1690-1650) in addition to the characteristic band of triazene (= 1444-1432) and $\mathrm{SO}_{2}$ group (= 13341314 and 1178-1138). ${ }^{1} \mathrm{H}-\mathrm{NMR}$ exhibited the presence of the piperazine protons $(=2.43-2.50$ and 3.15-4.00) together with the amino protons that disappeared on deuterium exchange together with the aromatic protons that was integrated for the corresponding compound (= 6.80-8.32). Mass spectra of compounds 11a and 14a revealed the molecular ion peaks of the compounds $(=726(\mathrm{M}-2)$ and 772 $\left.\left(\mathrm{M}^{+}\right)\right)$respectively).

Moreover, mild acid hydrolysis of 10a-e and 13a-e afforded compounds 12a-e and 15a-e respectively. IR spectra showed the appearance of $\mathrm{NH}$ band (=3427-3194), carbonyl band $(=1696-1654)$, in addition to the
Characteristic band of triazene (= 1444-1438) and $\mathrm{SO}_{2}$ group (= 13691320 and 1157-1142). ${ }^{1} \mathrm{H}-\mathrm{NMR}$ exhibited the presence of the piperazine protons $(=2.47-2.88$ and 3.31-3.40) together with the amino protons that disappeared on deuterium exchange and the aromatic protons that was integrated for the corresponding compound $(=6.81$ 8.54). Mass spectra of compounds 12b and 15a exhibited the molecular ion peaks of the compounds $(=566$ $(\mathrm{M}-2)$ and $538(\mathrm{M}+4))$ respectively).

Another method has been adopted for the synthesis of 12a via coupling of 9-chloroacridine-4-carbonyl chloride with anhydrous piperazine using the biphasic technique to yield 16 which was reacted with diazotized sulfanilamide to produce 12a Unfortunately, the yield of $\mathbf{1 6}$ was very low $(13 \%)$, which limited the usage of this pathway. IR of $\mathbf{1 6}$ showed the appearance of $\mathrm{NH}$ bands (3433, 3300), carbonyl band (= $1639)$, triazene band $(=1415)$ and $\mathrm{SO}_{2}$ bands $(=1338$ and 1152$) .{ }^{1} \mathrm{H}-$ NMR exhibited two triplets peaks for piperazine protons $(=2.81-2.93$ and $3.17-3.25)$, aromatic protons $(=7.24-$ 8.64) in addition to a singlet for the amino proton which disappeared on deuterium exchange $(=10.02)$. Finally, mass spectrum showed the molecular ion peaks of the compound $\left(=307\left(\mathrm{M}^{+}\right)\right)$. 

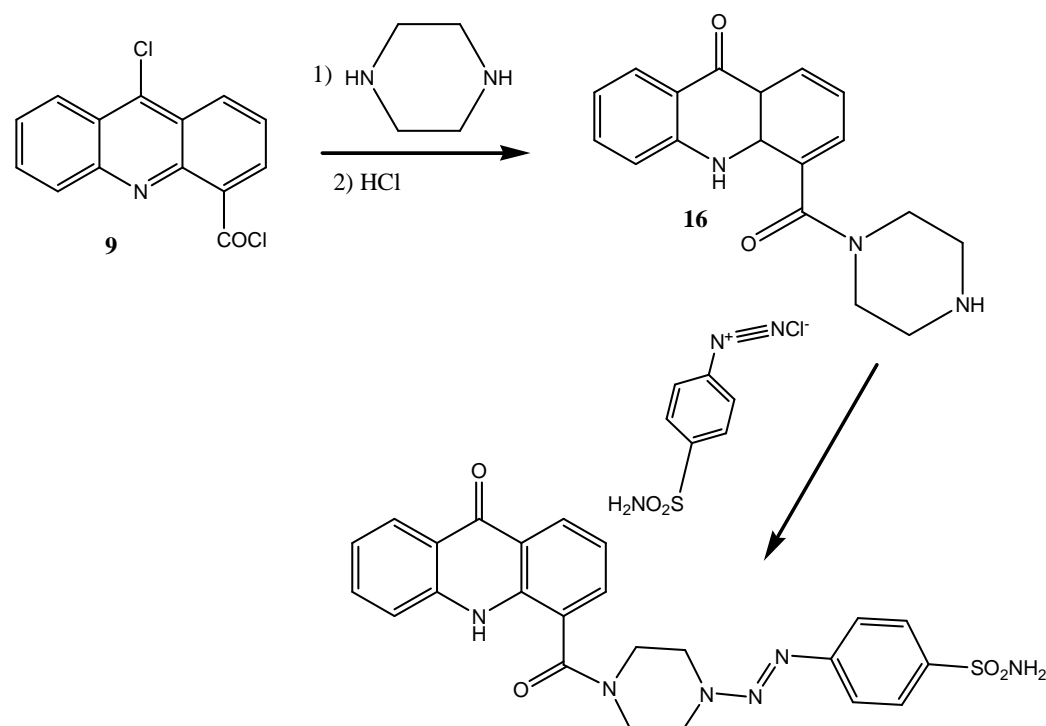

12
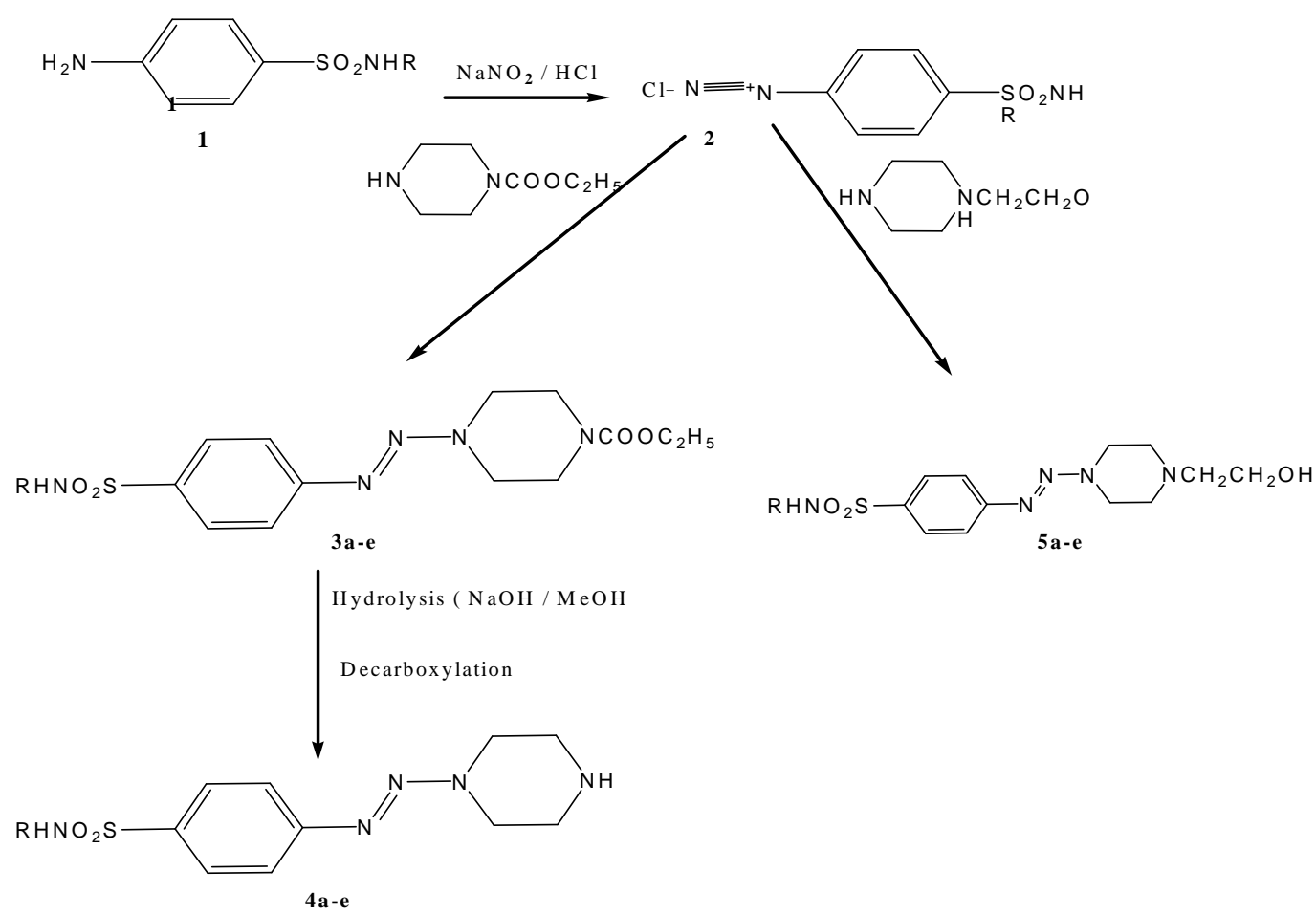

Scheme 1 


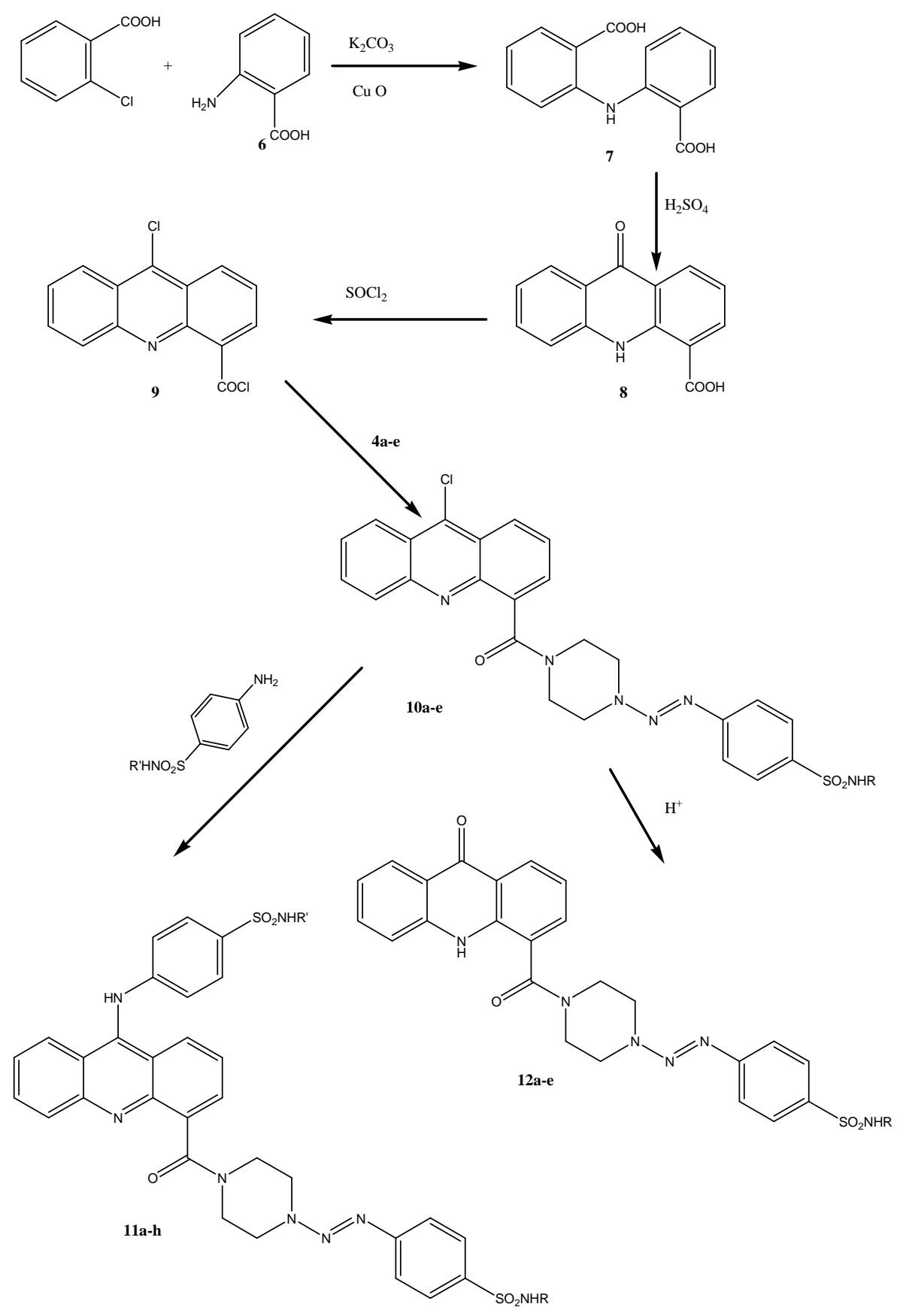

Scheme 2 

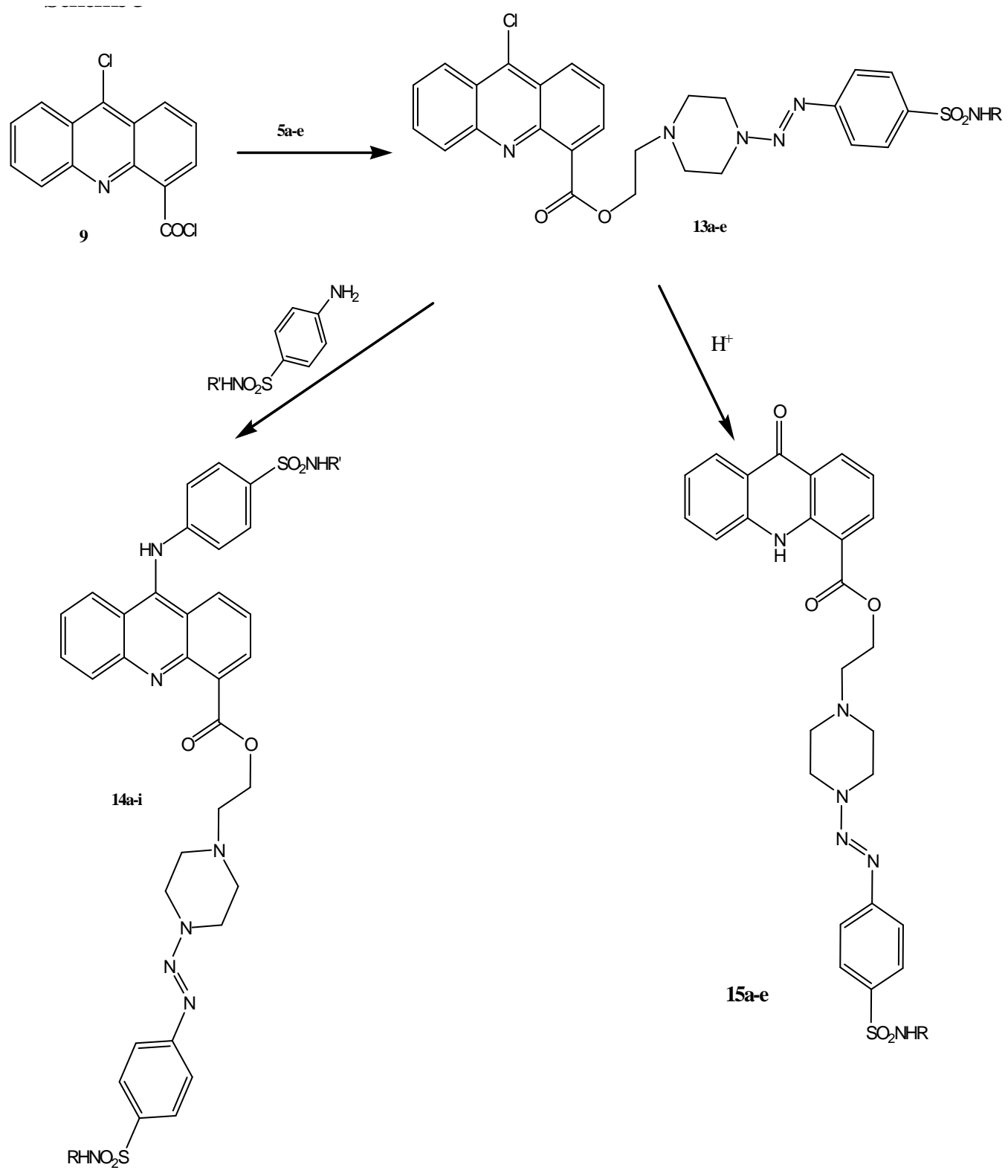

15a-e

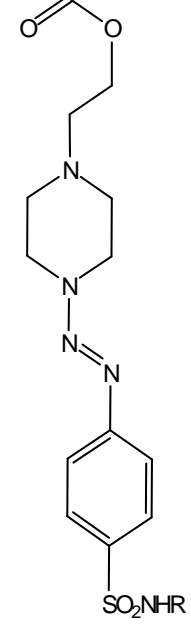

Scheme 3 


\section{EXPERIMENTAL}

\section{Chemistry}

All melting points were determined by the open capillary tube method using IA 9100MK-digital melting point apparatus and are uncorrected. IR spectra were recorded on Bruker Vector 22 (Germany)or Jasco FTIR plus 460 (Japan). ${ }^{1} \mathrm{H}-$ NMR spectra were recorded on a Varian Mercury VX-300 NMR spectrometer. ${ }^{1} \mathrm{H}-\mathrm{NMR}$. Spectra were run at $300 \mathrm{MHz}$ in deuterated chloroform $\left(\mathrm{CDCl}_{3}\right)$, dimethylsulfoxide (DMSO-d $\left.\mathrm{d}_{6}\right)$. Chemical shifts were quoted in $\delta$ units and were related to that of the solvents. Mass spectra were recorded on Finnigan MAT, SSQ 7000, mass spectrometer at $70 \mathrm{eV}$. Elemental Microanalysis were carried out using Heraew and Vario EL III (elemntar), CHNS analyzer (Germany) at the Microanalytical Center, Cairo University. Compounds $\mathbf{2}^{19}, \mathbf{3} \mathbf{a}^{19}, \mathbf{7}^{24}$, $\mathbf{8}^{4}$ and $\mathbf{9}^{4}$ were prepared according to the reported methods.

\section{Ethyl 4-((4-substituted sulfamoyl- phenyl)diazenyl)piperazine-1- carboxylates $3 b-e$}

The freshly prepared diazonium salt of 4-substituted sulfamoyl benzenediazonium chloride $\mathbf{2}$ (10 mmol) was neutralized with saturated aqueous sodium acetate solution $(\mathrm{pH}=6-7)$ in an ice bath $\left(0^{\circ} \mathrm{C}\right)$. Excess ethoxycarbonyl piperazine (2 $\mathrm{mL}, 12.6 \mathrm{mmol}$ ) was added to this stirred reaction mixture and stirring was continued for further 30 minutes while keeping the temperature at $0^{\circ} \mathrm{C}$, then neutralized with sodium acetate once more and left to stir for further an hour. The product was filtered, washed with water, dried and crystallized from hexane. ${ }^{1} \mathrm{H}-\mathrm{NMR}$ $\left(\right.$ DMSO- $\left._{6}\right)$ of $3 \mathrm{~d}: \delta=1.23(\mathrm{t}, 3 \mathrm{H}$, $\left.\mathrm{CH}_{2} \mathrm{CH}_{3}\right), 3.56$ (t, $4 \mathrm{H}$, piperazine $\mathrm{C}-3$, 5), 3.81 ( $\mathrm{t}, 4 \mathrm{H}$, piperazine $\mathrm{C}-2,6)$, 4.07-4.09 (q, 2H, $\mathrm{CH}_{2} \mathrm{CH}_{3}$ ), 6.81 (s, $4 \mathrm{H}, \mathrm{NH}_{2}, 2 \mathrm{NHs}\left(\mathrm{D}_{2} \mathrm{O}\right.$ exchange) $)$, $7.41(\mathrm{~d}, 2 \mathrm{H}$, aromatic $\mathrm{H} \mathrm{C}-2,6), 7.70$ (d, 2H, aromatic $\mathrm{H} \mathrm{C}-3,5)$. Mass spectrum of $\mathbf{3 d}: \mathrm{m} / \mathrm{z}(\%): \mathrm{M}+2=385$ $(4.21 \%)$.

\section{4-(Piperazine-1-yldiazenyl)subs- tituted benzenesulfonamides $4 a-e$}

Ethyl 4-((4-substituted sulfamoylphenyl)diazenyl)piperazine-1-

carboxylate 3a-e $(10 \mathrm{mmol})$ was refluxed with sodium hydroxide (1.2 $\mathrm{g}, 30 \mathrm{mmol})$ dissolved in methanol $(70 \mathrm{~mL})$, then the alcoholic layer was distilled off slowly during a two hour period. Heating was continued until the temperature of the residue was $130-135^{\circ} \mathrm{C}$ and left for 10 minutes at the same temperature in a sand bath. The residue was poured onto crushed ice and $\mathrm{pH}$ was adjusted to 7-8 using glacial acetic acid. The product was filtered, washed with water, dried and crystallized from $\mathrm{N}, \mathrm{N}$-dimethylformamide / water. ${ }^{1} \mathrm{H}-\mathrm{NMR}$ (DMSO$\left.\mathrm{d}_{6}\right)$ of $4 \mathbf{a}: \delta=2.49(\mathrm{t}, 4 \mathrm{H}$, piperazine $\mathrm{C}-3,5), 3.32(\mathrm{t}, 4 \mathrm{H}$, piperazine $\mathrm{C}-2$, 6), $7.28\left(\mathrm{~s}, 3 \mathrm{H}, \mathrm{NH}\right.$ and $\mathrm{NH}_{2}\left(\mathrm{D}_{2} \mathrm{O}\right.$ exchange) $), 7.48(\mathrm{~d}, 2 \mathrm{H}$, aromatic $\mathrm{H}$ C-2, 6), 7.78 (d, 2H, aromatic H C-3, 5). ${ }^{1}$ H-NMR (DMSO-d ${ }_{6}$ ) of $4 d: \delta=$ $2.48(\mathrm{t}, 4 \mathrm{H}$, piperazine $\mathrm{C}-3,5), 3.89$ (t, 4H, piperazine $\mathrm{C}-2,6), 7.03$ (s, $5 \mathrm{H}$, 
$3 \mathrm{NHs}$ and $\mathrm{NH}_{2}\left(\mathrm{D}_{2} \mathrm{O}\right.$ exchange $)$ ), 7.39-7.72 (m, 4H, aromatic $\mathrm{H})$. Mass spectrum of $4 \mathrm{a}: \mathrm{m} / \mathrm{z}(\%): \mathrm{M}^{+} 269$ $(2.73 \%)$.Mass spectrum of $\mathbf{4 d}: \mathrm{m} / \mathrm{z}$ (\%): $\mathrm{M}+2=313(29.57 \%)$.

\section{4-((4-(2-Hydroxyethyl)piperazine-} 1-yl)diazenyl)substituted benzenesulfonamides 5a-e

The freshly prepared diazonium salt of 4-substituted sulfamoylbenzenediazonium chloride $\mathbf{2}$ (10 mmol) was neutralized with saturated aqueous sodium acetate solution $(\mathrm{pH}=6-7)$ in an ice bath $\left(0^{\circ} \mathrm{C}\right)$. Excess hydroxyethyl piperazine (1.6 $\mathrm{mL}, 12.6 \mathrm{mmol}$ ) was added to this stirred reaction mixture and stirring was continued for further 30 minutes in an ice bath, then neutralized with sodium acetate once more and left to stir for further an hour. The product was filtered, washed with water, dried and crystallized from N,Ndimethylformamide / water. ${ }^{1} \mathrm{H}-\mathrm{NMR}$ $\left(\right.$ DMSO-d $\left._{6}\right)$ of 5a: $\delta=2.49$ (t, $4 \mathrm{H}$, piperazine $\mathrm{C}-3,5), 2.72(\mathrm{~s}, 2 \mathrm{H}$, $\left.\mathrm{OCH}_{2} \underline{\mathrm{CH}}_{2}\right), 2.88$ (s, $2 \mathrm{H}, \mathrm{OCH}_{2} \mathrm{CH}_{2}$ ), $3.26(\mathrm{t}, 4 \mathrm{H}$, piperazine $\mathrm{C}-2,6), 3.7$ (s, $1 \mathrm{H}, \mathrm{OH}\left(\mathrm{D}_{2} \mathrm{O}\right.$ exchange $\left.)\right), 7.29-7.92$ $(\mathrm{m}, 4 \mathrm{H}$, aromatic $\mathrm{H}), 7.94(\mathrm{~s}, 2 \mathrm{H}$, $\mathrm{NH}_{2} \quad\left(\mathrm{D}_{2} \mathrm{O}\right.$ exchange $)$. ${ }^{1} \mathrm{H}-$ NMR(DMSO- $\left.\mathrm{d}_{6}\right)$ of $\mathbf{5 d}: \delta=2.43(\mathrm{t}$, $4 \mathrm{H}$, piperazine $\mathrm{C}-3,5), 2.57$ (t, $2 \mathrm{H}$, $\left.\mathrm{OCH}_{2} \mathrm{CH}_{2}\right), 3.35$ (t, $2 \mathrm{H}, \mathrm{OCH}_{2} \mathrm{CH}_{2}$ ), $3.73(\mathrm{t}, 4 \mathrm{H}$, piperazine $\mathrm{C}-2,6), 4.01$ (s, $1 \mathrm{H}, \mathrm{OH}\left(\mathrm{D}_{2} \mathrm{O}\right.$ exchange) $), 6.84$ (s, $4 \mathrm{H}, 2 \mathrm{NHs}$ and $\mathrm{NH}_{2}\left(\mathrm{D}_{2} \mathrm{O}\right.$ exchange) $)$, $7.37(\mathrm{~d}, 2 \mathrm{H}$, aromatic $\mathrm{H} \mathrm{C}-3,5), 7.67$ (d, 2H, aromatic $\mathrm{H} \mathrm{C}-2,6)$. Mass spectrum of $5 \mathbf{a}: \mathrm{m} / \mathrm{z}(\%): \mathrm{M}^{+} 313$ $(11.93 \%)$.
4-((4-(9-Chloroacridine-4-carbonyl) piperazin-1-yl)diazenyl)-N-substituted benzenesulfonamides 10a-e

Freshly prepared 9-chloroacridine4-carbonyl chloride 9 obtained from acridone-4-carboxylic acid 8 (1 g, $4.18 \mathrm{mmol}$ ) was dissolved in dry $\mathrm{N}, \mathrm{N}$-dimethylformamide $(5 \mathrm{~mL})$ and then added to a solution of 4a-e (4.18 mmol) in dry N,N-dimethylformamide $(5 \mathrm{~mL})$ catalyzed with triethylamine $(0.5 \mathrm{~mL})$. The mixture was heated in a water bath for an hour. The resulting solution was used directly in the next step due to its instability.

N-(4-(4-((4-Substituted sulfamoyl)phenyl)diazenyl)piperazine-1carbonyl) acridin-9-ylamino)substituted benzene sulfonamides 11a-h

The appropriate sulfonamide (4.18 mmol) was dissolved in dry $\mathrm{N}, \mathrm{N}$ dimethylformamide $(5 \mathrm{~mL})$, acidified with dilute hydrochloric acid (3 drops), added to the stirred mixture of the corresponding 10a-e, and left to stir in a water bath for another one hour. The reaction mixture was diluted with ice-water and dilute ammonium hydroxide solution was added till complete precipitation $(\mathrm{pH}=7)$. The product was filtered, washed with water, dried and crystallized from N,N-dimethylformamide / water. ${ }^{1} \mathrm{H}$-NMR (DMSO$\left.\mathrm{d}_{6}\right)$ of 11a: $\delta=2.43-2.49(\mathrm{~m}, 8 \mathrm{H}$, piperazine $\mathrm{H}), 2.88\left(\mathrm{~s}, 3 \mathrm{H}, \mathrm{COCH}_{3}\right)$, $6.76\left(\mathrm{~s}, 2 \mathrm{H}, 2 \mathrm{NHs}\left(\mathrm{D}_{2} \mathrm{O}\right.\right.$ exchange) , 6.99 (s, $2 \mathrm{H}, \mathrm{NH}_{2}\left(\mathrm{D}_{2} \mathrm{O}\right.$ exchange) $)$ 7.31-8.32 (m, 15H, aromatic $\mathrm{H}), 8.43$ 
(s, 1H, NH ( $\mathrm{D}_{2} \mathrm{O}$ exchange)), 10.87 (s, $1 \mathrm{H}, \mathrm{NH}\left(\mathrm{D}_{2} \mathrm{O}\right.$ exchange) $) .{ }^{1} \mathrm{H}-$ NMR (DMSO-d ${ }_{6}$ )of 11b: $\delta=2.24(\mathrm{~s}$, $\left.3 \mathrm{H}, \mathrm{CH}_{3}\right), 2.57\left(\mathrm{~s}, 3 \mathrm{H}, \mathrm{CH}_{3}\right), 2.73$ (s, $4 \mathrm{H}$, piperazine $\mathrm{C}-2,6), 2.88$ (s, $4 \mathrm{H}$, piperazine C-3, 5), 6.60-8.55 (m, $17 \mathrm{H}$, aromatic $\mathrm{H}$ and $\mathrm{NH}$ ), 10.95 (s, $\left.2 \mathrm{H}, \mathrm{SO}_{2} \mathrm{NH}_{2}\right), 11.60\left(\mathrm{~s}, 1 \mathrm{H}, \mathrm{SO}_{2} \mathrm{NH}\right)$. ${ }^{1} \mathrm{H}-\mathrm{NMR}$ (DMSO-d $\left.\mathrm{d}_{6}\right)$ of $\mathbf{1 1 h}: \delta=$ 2.72-3.15 (m, 8H, piperazine $\mathrm{H}$ ), 7.36 (s, $4 \mathrm{H}, 2 \mathrm{NHs}, \mathrm{NH}_{2}\left(\mathrm{D}_{2} \mathrm{O}\right.$ exchange)), 7.56-8.17 (m, 18H, aromatic $\mathrm{H})$. Mass spectrum 11a: $\mathrm{m} / \mathrm{z}(\%): \mathrm{M}-2=$ $726(12.75 \%)$.

\section{4-((4-(9-Oxo-9,10-dihydroacridine- 4-carbonyl)piperazine-1-yl)- diazenyl)-N-substituted benzene- sulfonamides 12a-e}

The stirred reaction mixture of 10a-e was acidified with dilute hydrochloric acid $(5 \mathrm{~mL})$, and left to stir in a water bath for another hour. The reaction mixture was diluted with ice-water and dilute ammonium hydroxide solution was added till complete precipitation $(\mathrm{pH}=7)$. The product was filtered, washed with water, dried and crystallized from $\mathrm{N}$,N-dimethylformamide / $\mathrm{H}_{2} \mathrm{O} .{ }^{1} \mathrm{H}-$ NMR (DMSO- $\left.\mathrm{d}_{6}\right)$ of 12a: $\delta=2.72(\mathrm{t}$, $4 \mathrm{H}$, piperazine $\mathrm{C}-2,6), 2.88(\mathrm{t}, 4 \mathrm{H}$, piperazine $\mathrm{C}-3,5), 6.81-8.41(\mathrm{~m}$, $14 \mathrm{H}$, aromatic $\mathrm{H}), 8.50(\mathrm{~s}, 1 \mathrm{H}, \mathrm{NH}$ $\left(\mathrm{D}_{2} \mathrm{O}\right.$ exchange $\left.)\right), 8.54(\mathrm{~s}, 1 \mathrm{H}, \mathrm{NH}$ ( $\mathrm{D}_{2} \mathrm{O}$ exchange)). ${ }^{1} \mathrm{H}-\mathrm{NMR}$ (TFAA) of $12 \mathrm{~d}$ : $3.15(\mathrm{t}, 4 \mathrm{H}$, piperazine $\mathrm{C}-2$, 6), $3.23(\mathrm{t}, 4 \mathrm{H}$, piperazine $\mathrm{C}-3,5)$, 7.82-9.12 $(\mathrm{m}, 16 \mathrm{H}, 11$ aromatic $\mathrm{H}$, $3 \mathrm{NHs}$ and $\mathrm{NH}_{2}$ ). ${ }^{1} \mathrm{H}-\mathrm{NMR}$ (DMSO$\left.\mathrm{d}_{6}\right)$ of 12e: $\delta=2.70(\mathrm{t}, 4 \mathrm{H}$, piperazine $\mathrm{C}-2,6), 2.92$ (s, 3H, $\left.\mathrm{COCH}_{3}\right), 3.01$ (t,
$4 \mathrm{H}$, piperazine $\mathrm{C}-3$, 5), 7.20-8.30 (m, $11 \mathrm{H}$, aromatic $\mathrm{H}), 10.70(\mathrm{~s}, 1 \mathrm{H}, \mathrm{NH}$ $\left(\mathrm{D}_{2} \mathrm{O}\right.$ exchange $\left.)\right), 10.80(\mathrm{~s}, 1 \mathrm{H}, \mathrm{NH}$ $\left(\mathrm{D}_{2} \mathrm{O}\right.$ exchange)). Mass spectrum of 12b: $\mathrm{m} / \mathrm{z}(\%): \mathrm{M}-2=566(16.36 \%)$.

\section{2-(4-((4-N-substitutedsulfamoyl- phenyl)diazenyl)piperazin-1-yl)- ethyl-9-chloroacridin-4- carboxylates 13a-e}

Freshly prepared 9-chloroacridine4-carbonyl chloride 9 obtained from acridone-4-carboxylic acid 8 (1 g, $4.18 \mathrm{mmol}$ ) was dissolved in dry $\mathrm{N}, \mathrm{N}$-dimethylformamide $(5 \mathrm{~mL})$ and then added to a solution of the appropriate 5a-e $(4.18 \mathrm{mmol})$ in dry $\mathrm{N}, \mathrm{N}$-dimethylformamide $(5 \mathrm{~mL})$ catalyzed with triethylamine $(0.5$ $\mathrm{mL}$ ). The mixture was heated in a water bath for an hour. The resulting solution was used directly in the next step due to its instability.

\section{2-(4-((4-N-substitutedsulfamoyl)- phenyl)diazenyl)piperazin-1-yl)- ethyl-9-(4-N-substituted sulfamoyl)- phenylamino)-acridine-4-carboxy- late 14a-j}

The appropriate sulfonamide (4.18 mmol) was dissolved in dry $\mathrm{N}, \mathrm{N}$ dimethylformamide $(5 \mathrm{~mL})$, acidified with dilute hydrochloric acid (3 drops), added to the stirred mixture of 13a-e and stirring was continued in a water bath for another hour. The reaction mixture was diluted with icewater and dilute ammonium hydroxide solution was added till complete precipitation $(\mathrm{pH}=7)$. The product was filtered, washed with water, dried and crystallized from $\mathrm{N}, \mathrm{N}$-dimethylformamide / water. ${ }^{1} \mathrm{H}-$ 
NMR (DMSO- $\left.\mathrm{d}_{6}\right)$ of 14a: $\delta=2.49(\mathrm{t}$, $4 \mathrm{H}$, piperazine $\mathrm{C}-2,6), 2.65(\mathrm{t}, 2 \mathrm{H}$ $\left.\mathrm{OCH}_{2} \mathrm{CH}_{2}\right), 2.85$ (t, $2 \mathrm{H}, \mathrm{OCH}_{2} \mathrm{CH}_{2}$ ), $3.59(\mathrm{t}, 4 \mathrm{H}$, piperazine $\mathrm{C}-3,5)$, $6.77\left(\mathrm{~s}, 7 \mathrm{H}, 3 \mathrm{NHs}\right.$ and $2 \mathrm{NH}_{2} \mathrm{~s}\left(\mathrm{D}_{2} \mathrm{O}\right.$ exchange) $), \quad 7.28-8.47 \quad(\mathrm{~m}, \quad 15 \mathrm{H}$, aromatic $\mathrm{H}), 10.90(\mathrm{~s}, 2 \mathrm{H}, 2 \mathrm{NHs}$ $\left(\mathrm{D}_{2} \mathrm{O}\right.$ exchange)). ${ }^{1} \mathrm{H}-\mathrm{NMR}$ (DMSO$\left.\mathrm{d}_{6}\right)$ of 14d: $\delta=2.49(\mathrm{t}, 4 \mathrm{H}$, piperazine $\mathrm{C}-2,6), 2.71$ (t, $\left.2 \mathrm{H}, \mathrm{OCH}_{2} \mathrm{CH}_{2}\right), 2.87$ $\left(\mathrm{t}, 2 \mathrm{H}, \quad \mathrm{OCH}_{2} \mathrm{CH}_{2}\right), 4.00 \quad(\mathrm{t}, 4 \mathrm{H}$, piperazine $\mathrm{C}-3,5), 6.80-8.53(\mathrm{~m}$, $20 \mathrm{H}, 18$ aromatic $\mathrm{H}$ and 2NHs), 9.50 (s, $2 \mathrm{H}, \mathrm{NH}_{2}$ ). ${ }^{1} \mathrm{H}-\mathrm{NMR}$ (DMSO-d $\mathrm{d}_{6}$ ) of 14f: $\delta=2.48(\mathrm{t}, 4 \mathrm{H}$, piperazine $\mathrm{C}-2$, 6), $2.72\left(\mathrm{t}, 2 \mathrm{H}, \mathrm{OCH}_{2} \mathrm{CH}_{2}\right), 2.88$ (t, $\left.2 \mathrm{H}, \quad \mathrm{OCH}_{2} \mathrm{CH}_{2}\right), \quad 3.45 \quad(\mathrm{t}, \quad 4 \mathrm{H}$, piperazine $\mathrm{C}-3,5), 7.34-8.50 \quad(\mathrm{~m}$, $22 \mathrm{H}, 18$ aromatic $\mathrm{H}, 2 \mathrm{NHs}$ and $\mathrm{NH}_{2}$ $\left(\mathrm{D}_{2} \mathrm{O}\right.$ exchange)). Mass spectrum of 14a: $\mathrm{m} / \mathrm{z}(\%): \mathrm{M}^{+} 772(0.13 \%)$.

\section{2-(4-((4-N-substitutedsulfamoyl- phenyl)diazenyl)piperazin-1- yl)ethyl-9-oxo-9,10-dihydro- acridine-4-carboxylate 15a-e}

The stirred reaction mixture of the appropriate 13a-e was acidified with dilute hydrochloric acid $(5 \mathrm{~mL})$, and left to stir in a water bath for another hour. The reaction mixture was diluted with ice-water and dilute ammonium hydroxide solution was added till complete precipitation $(\mathrm{pH}$ $=7$ ). The product was filtered, washed with water, dried and crystallized from N,N-dimethylformamide / water. ${ }^{1} \mathrm{H}$-NMR (DMSO$\left.\mathrm{d}_{6}\right)$ of 15a: $\delta=2.49(\mathrm{t}, 4 \mathrm{H}$, piperazine C-2, 6), 2.70 (t, 2H, $\mathrm{OCH}_{2} \mathrm{CH}_{2}$ ), 2.90 $\left(\mathrm{t}, 2 \mathrm{H}, \quad \mathrm{OCH}_{2} \mathrm{CH}_{2}\right), 3.41(\mathrm{t}, 4 \mathrm{H}$, piperazine $\mathrm{C}-3,5), \quad 7.30-8.52(\mathrm{~m}$, $14 \mathrm{H}, 11$ aromatic $\mathrm{H}, \mathrm{NH}$ and $\mathrm{NH}_{2}$ $\left(\mathrm{D}_{2} \mathrm{O}\right.$ exchange)). ${ }^{1} \mathrm{H}-\mathrm{NMR}$ (TFAA) of 15d: $\delta=2.19$ (t, $4 \mathrm{H}$, piperazine $\mathrm{C}$ 2, 6), $2.30\left(\mathrm{t}, 2 \mathrm{H}, \mathrm{OCH}_{2} \mathrm{CH}_{2}\right), 3.10$ (t, $\left.2 \mathrm{H}, \quad \mathrm{OCH}_{2} \mathrm{CH}_{2}\right), \quad 3.30 \quad(\mathrm{t}, \quad 4 \mathrm{H}$, piperazine $\mathrm{C}-3,5), 7.61-9.08 \quad(\mathrm{~m}$, $20 \mathrm{H}, 15$ aromatic $\mathrm{H}, 3 \mathrm{NHs}$ and $\mathrm{NH}_{2}$ ( $\mathrm{D}_{2} \mathrm{O}$ exchange)). Mass spectrum of 15a: $\mathrm{m} / \mathrm{z}(\%): \mathrm{M}^{+}+4=538(0.9 \%)$.

\section{Biological screening}

Fourteen representative compounds have been chosen and tested for their cytotoxic activity against human mammary carcinoma cell line in the National Cancer Institute, Cairo University. The screening involves calculation of the percentage growth of surviving fraction of the drug treated cell lines compared by untreated control using Sulforhodamie B (SRB) colorimetric assay. Sulforhodamie B is a bright pink aminoxanthene anionic dye with two sulfonic acid groups that bind electrostatically to protein basic amino acid residues of trichloroacetic acid fixed cells under mild acidic conditions $^{25}$. Cultures fixed with trichlroacetic acid were stained for 30 minutes with $0.4 \% \quad \mathrm{w} / \mathrm{v}$ Sulforhodamie B dissolved in $1 \%$ acetic acid, and protein-bound dye was extracted with $10 \mathrm{mM}$ tris base for determination of optical density in a computer-interfaced, 96-well microtiter plate reader.

The optical density measured is linear to the cell number of the surviving fraction. Therefore, the 
Table 1: Physical, microanalytical and I.R. data of compounds 3b-e, 4a-e, 5a-e, 12a-e and 15a-e.

\begin{tabular}{|c|c|c|c|c|c|c|}
\hline \multirow{2}{*}{$\begin{array}{l}\text { Cpd. } \\
\text { No. }\end{array}$} & \multirow{2}{*}{$\mathrm{R}$} & \multirow{2}{*}{$\begin{array}{l}\text { Molecular } \\
\text { Formula } \\
\text { M. Wt. } \\
\end{array}$} & \multirow{2}{*}{$\begin{array}{c}\text { Yield } \\
(\%) \\
\text { M.P. }\left({ }^{\circ} \mathrm{C}\right) \\
\end{array}$} & \multicolumn{2}{|c|}{ Microanalysis (\%) } & \multirow{2}{*}{$\operatorname{IR}\left(\mathrm{cm}^{-1}\right)$} \\
\hline & & & & Calc. & Found & \\
\hline $3 b$ & & $\begin{array}{c}\mathrm{C}_{17} \mathrm{H}_{21} \mathrm{~N}_{7} \mathrm{O}_{4} \mathrm{~S} \\
419.46\end{array}$ & $\begin{array}{c}59 \\
230-2\end{array}$ & $\begin{array}{l}\text { C } 48.67 \\
\text { H } 5.04 \\
\text { N } 23.37 \\
\end{array}$ & $\begin{array}{c}48.90 \\
5.01 \\
23.38 \\
\end{array}$ & $\begin{array}{l}3396(\mathrm{NHs}), 1655(\mathrm{CO}), \\
1436(\mathrm{~N}=\mathrm{N}-\mathrm{N}), 1340, \\
1159\left(\mathrm{SO}_{2}\right) .\end{array}$ \\
\hline $3 c$ & & $\begin{array}{c}\mathrm{C}_{19} \mathrm{H}_{25} \mathrm{~N}_{7} \mathrm{O}_{4} \mathrm{~S} \\
447.51\end{array}$ & $\begin{array}{c}56 \\
200-2\end{array}$ & $\begin{array}{l}\text { C } 50.99 \\
\text { H } 5.63 \\
\text { N } 21.90 \\
\end{array}$ & $\begin{array}{c}51.19 \\
5.31 \\
22.21 \\
\end{array}$ & $\begin{array}{l}3447(\mathrm{NH}), 1652(\mathrm{CO}) \\
1431(\mathrm{~N}=\mathrm{N}-\mathrm{N}), 1350, \\
1150\left(\mathrm{SO}_{2}\right)\end{array}$ \\
\hline 3d & & $\begin{array}{c}\mathrm{C}_{14} \mathrm{H}_{21} \mathrm{~N}_{7} \mathrm{O}_{4} \mathrm{~S} \\
383.14\end{array}$ & $\begin{array}{c}65 \\
235-7\end{array}$ & $\begin{array}{l}\text { C } 43.85 \\
\text { H } 5.52 \\
\text { N } 25.57\end{array}$ & $\begin{array}{c}44.06 \\
5.33 \\
25.66\end{array}$ & $\begin{array}{l}3445,3339,3203\left(\mathrm{NH}_{2},\right. \\
\mathrm{NHs}), 1683(\mathrm{CO}), 1427 \\
(\mathrm{~N}=\mathrm{N}-\mathrm{N}), 1350,1133 \\
\left(\mathrm{SO}_{2}\right) .\end{array}$ \\
\hline $3 e$ & & $\begin{array}{c}\mathrm{C}_{15} \mathrm{H}_{21} \mathrm{~N}_{5} \mathrm{O}_{5} \mathrm{~S} \\
383.42\end{array}$ & $\begin{array}{c}53 \\
215-17\end{array}$ & $\begin{array}{c}\text { C } 46.98 \\
\text { H } 5.52 \\
\text { N } 18.26 \\
\end{array}$ & $\begin{array}{c}46.30 \\
5.12 \\
18.30 \\
\end{array}$ & $\begin{array}{l}3311(\mathrm{NH}), 1688(\mathrm{CO}) \\
1439(\mathrm{~N}=\mathrm{N}-\mathrm{N}), 1338 \\
1149\left(\mathrm{SO}_{2}\right)\end{array}$ \\
\hline $4 a$ & $\mathrm{H}$ & $\begin{array}{c}\mathrm{C}_{10} \mathrm{H}_{15} \mathrm{~N}_{5} \mathrm{O}_{2} \mathrm{~S} \\
269.32\end{array}$ & $\begin{array}{c}58 \\
255-7\end{array}$ & $\begin{array}{c}\text { C } 44.59 \\
\text { H } 5.61 \\
\text { N } 26.00 \\
\end{array}$ & $\begin{array}{c}44.55 \\
5.50 \\
25.90 \\
\end{array}$ & $\begin{array}{l}3080,3060\left(\mathrm{NH}_{2}, \mathrm{NH}\right) \\
1420(\mathrm{~N}=\mathrm{N}-\mathrm{N}) ; 1320 \\
1150\left(\mathrm{SO}_{2}\right)\end{array}$ \\
\hline $4 b$ & & $\begin{array}{c}\mathrm{C}_{14} \mathrm{H}_{17} \mathrm{~N}_{7} \mathrm{O}_{2} \mathrm{~S} \\
347.39\end{array}$ & $\begin{array}{c}66 \\
155-7\end{array}$ & $\begin{array}{l}\text { C } 48.40 \\
\text { H } 4.93 \\
\text { N } 28.22 \\
\end{array}$ & $\begin{array}{c}48.30 \\
5.20 \\
28.12 \\
\end{array}$ & $\begin{array}{l}3400,3298(\mathrm{NHs}), 1447 \\
(\mathrm{~N}=\mathrm{N}-\mathrm{N}), 1355,1154 \\
\left(\mathrm{SO}_{2}\right) .\end{array}$ \\
\hline $4 c$ & & $\begin{array}{c}\mathrm{C}_{16} \mathrm{H}_{21} \mathrm{~N}_{7} \mathrm{O}_{2} \mathrm{~S} \\
375.45\end{array}$ & $\begin{array}{c}64 \\
109-11\end{array}$ & $\begin{array}{l}\text { C } 51.18 \\
\text { H } 5.63 \\
\text { N } 26.11 \\
\end{array}$ & $\begin{array}{l}\text { C } 51.10 \\
\text { H } 5.50 \\
\text { N } 25.56 \\
\end{array}$ & $\begin{array}{l}3100(\mathrm{NH}), 1440(\mathrm{~N}=\mathrm{N}- \\
\mathrm{N}), 1320,1140\left(\mathrm{SO}_{2}\right)\end{array}$ \\
\hline 4d & & $\begin{array}{c}\mathrm{C}_{11} \mathrm{H}_{17} \mathrm{~N}_{7} \mathrm{O}_{2} \mathrm{~S} \\
311.11\end{array}$ & $\begin{array}{c}70 \\
182-4\end{array}$ & $\begin{array}{l}\text { C } 42.43 \\
\text { H } 5.50 \\
\text { N } 31.48 \\
\end{array}$ & $\begin{array}{l}\text { C } 42.26 \\
\text { H } 5.80 \\
\text { N } 31.78 \\
\end{array}$ & $\begin{array}{l}3446,3339,3200\left(\mathrm{NH}_{2},\right. \\
\mathrm{NHs}), 1427(\mathrm{~N}=\mathrm{N}-\mathrm{N}), \\
1349,1133\left(\mathrm{SO}_{2}\right) .\end{array}$ \\
\hline $4 e$ & & $\begin{array}{c}\mathrm{C}_{12} \mathrm{H}_{17} \mathrm{~N}_{5} \mathrm{O}_{3} \mathrm{~S} \\
311.36\end{array}$ & $\begin{array}{c}62 \\
154-6\end{array}$ & $\begin{array}{c}\text { C } 46.29 \\
\text { H } 5.50 \\
\text { N } 20.39 \\
\end{array}$ & $\begin{array}{c}46.03 \\
5.93 \\
20.15 \\
\end{array}$ & $\begin{array}{l}3313,3243(\mathrm{NHs}), 1687 \\
(\mathrm{CO}), 1436(\mathrm{~N}=\mathrm{N}-\mathrm{N}), \\
1338,1152\left(\mathrm{SO}_{2}\right) .\end{array}$ \\
\hline $5 a$ & $\mathbf{H}$ & $\begin{array}{c}\mathrm{C}_{12} \mathrm{H}_{19} \mathrm{~N}_{5} \mathrm{O}_{3} \mathrm{~S} \\
313.37\end{array}$ & $\begin{array}{c}56 \\
290-1\end{array}$ & $\begin{array}{l}\text { C } 45.99 \\
\text { H } 6.11 \\
\text { N } 22.34\end{array}$ & $\begin{array}{c}45.80 \\
6.10 \\
22.32\end{array}$ & $\begin{array}{l}3419(\mathrm{OH}), 3375\left(\mathrm{NH}_{2},\right. \\
\mathrm{NHs}), 1438(\mathrm{~N}=\mathrm{N}-\mathrm{N}) \\
1334,1156\left(\mathrm{SO}_{2}\right)\end{array}$ \\
\hline
\end{tabular}


Table 1: Continued.

\begin{tabular}{|c|c|c|c|c|c|c|}
\hline \multirow{2}{*}{$\begin{array}{l}\text { Cpd. } \\
\text { No. }\end{array}$} & \multirow{2}{*}{$\mathrm{R}$} & \multirow{2}{*}{$\begin{array}{l}\text { Molecular } \\
\text { Formula } \\
\text { M. Wt. }\end{array}$} & \multirow{2}{*}{$\begin{array}{c}\text { Yield } \\
(\%) \\
\text { M.P. }\left({ }^{\circ} \mathrm{C}\right) \\
\end{array}$} & \multicolumn{2}{|c|}{ Microanalysis (\%) } & \multirow{2}{*}{$\operatorname{IR}\left(\mathrm{cm}^{-1}\right)$} \\
\hline & & & & Calc. & Found & \\
\hline $5 \mathbf{b}$ & & $\begin{array}{c}\mathrm{C}_{16} \mathrm{H}_{21} \mathrm{~N}_{7} \mathrm{O}_{3} \mathrm{~S} \\
391.45\end{array}$ & $\begin{array}{c}68 \\
252-4\end{array}$ & $\begin{array}{l}\text { C } 49.09 \\
\text { H } 5.40 \\
\text { N } 25.04\end{array}$ & $\begin{array}{c}48.58 \\
5.73 \\
25.04 \\
\end{array}$ & $\begin{array}{l}3446(\mathrm{OH}), 3279(\mathrm{NH}), \\
1612,1422(\mathrm{~N}=\mathrm{N}-\mathrm{N}), \\
1311,1153\left(\mathrm{SO}_{2}\right)\end{array}$ \\
\hline $5 c$ & & $\begin{array}{c}\mathrm{C}_{18} \mathrm{H}_{25} \mathrm{~N}_{7} \mathrm{O}_{3} \mathrm{~S} \\
419.50\end{array}$ & $\begin{array}{c}65 \\
200-2\end{array}$ & $\begin{array}{l}\text { C } 51.53 \\
\text { H } 6.00 \\
\text { N } 23.37\end{array}$ & $\begin{array}{c}51.29 \\
6.23 \\
23.37\end{array}$ & $\begin{array}{l}3444(\mathrm{OH}, \mathrm{NH}), 1428 \\
(\mathrm{~N}=\mathrm{N}-\mathrm{N}), 1308,1147 \\
\left(\mathrm{SO}_{2}\right) .\end{array}$ \\
\hline $5 d$ & & $\begin{array}{c}\mathrm{C}_{13} \mathrm{H}_{21} \mathrm{~N}_{7} \mathrm{O}_{3} \mathrm{~S} \\
355.41\end{array}$ & $\begin{array}{c}72 \\
216-8\end{array}$ & $\begin{array}{l}\text { C } 43.93 \\
\text { H } 5.95 \\
\text { N } 27.58\end{array}$ & $\begin{array}{c}43.81 \\
5.73 \\
27.68 \\
\end{array}$ & $\begin{array}{l}3448,3371,3207(\mathrm{OH}, \\
\left.\mathrm{NH}_{2}, \mathrm{NHs}\right), 1433(\mathrm{~N}=\mathrm{N}- \\
\mathrm{N}), 1363,1132\left(\mathrm{SO}_{2}\right) .\end{array}$ \\
\hline $5 e$ & & $\begin{array}{c}\mathrm{C}_{14} \mathrm{H}_{21} \mathrm{~N}_{5} \mathrm{O}_{4} \mathrm{~S} \\
355.41\end{array}$ & $\begin{array}{c}59 \\
155-7\end{array}$ & $\begin{array}{l}\text { C } 47.31 \\
\text { H } 5.95 \\
\text { N } 19.70\end{array}$ & $\begin{array}{c}47.10 \\
5.90 \\
19.60\end{array}$ & $\begin{array}{l}3368(\mathrm{OH}), 3291(\mathrm{NH}), \\
1436(\mathrm{~N}=\mathrm{N}-\mathrm{N}), 1330, \\
1156\left(\mathrm{SO}_{2}\right)\end{array}$ \\
\hline $12 a$ & $\overline{\mathrm{H}}$ & $\begin{array}{c}\mathrm{C}_{24} \mathrm{H}_{22} \mathrm{~N}_{6} \mathrm{O}_{4} \mathrm{~S} \\
490.14\end{array}$ & $\begin{array}{c}60 \\
290-2\end{array}$ & $\begin{array}{c}\text { C } 58.76 \\
\text { H } 4.52 \\
\text { N } 17.13 \\
\end{array}$ & $\begin{array}{c}\text { C } 58.85 \\
\text { H } 4.31 \\
\text { N } 17.08 \\
\end{array}$ & $\begin{array}{l}3359,3258\left(\mathrm{NH}_{2}, \mathrm{NHs}\right), \\
1686(\mathrm{CO}), 1440(\mathrm{~N}=\mathrm{N}- \\
\mathrm{N}), 1369,1142\left(\mathrm{SO}_{2}\right) .\end{array}$ \\
\hline $12 \mathrm{~b}$ & & $\begin{array}{c}\mathrm{C}_{28} \mathrm{H}_{24} \mathrm{~N}_{8} \mathrm{O}_{4} \mathrm{~S} \\
568.60\end{array}$ & $\begin{array}{c}72 \\
278-9\end{array}$ & $\begin{array}{l}\text { C } 59.14 \\
\text { H } 4.25 \\
\text { N } 19.70\end{array}$ & $\begin{array}{c}\text { C } 59.01 \\
\mathrm{H} 4.54 \mathrm{~N} \\
19.60 \\
\end{array}$ & $\begin{array}{l}3261,3194 \text { (NHs), } 1690 \\
(\mathrm{CO}), 1444(\mathrm{~N}=\mathrm{N}-\mathrm{N}), \\
1320,1150\left(\mathrm{SO}_{2}\right) .\end{array}$ \\
\hline $14 \mathrm{c}$ & & $\begin{array}{c}\mathrm{C}_{30} \mathrm{H}_{28} \mathrm{~N}_{8} \mathrm{O}_{4} \mathrm{~S} \\
596.66\end{array}$ & $\begin{array}{c}70 \\
222-4\end{array}$ & $\begin{array}{l}\text { C } 60.39 \\
\text { H } 4.73 \\
\text { N } 18.78\end{array}$ & $\begin{array}{c}60.50 \\
4.90 \\
18.85\end{array}$ & $\begin{array}{l}3418,3294 \text { (NHs), } 1690 \\
(\mathrm{CO}), 1438(\mathrm{~N}=\mathrm{N}-\mathrm{N}), \\
1330,1152\left(\mathrm{SO}_{2}\right) .\end{array}$ \\
\hline $12 d$ & & $\begin{array}{c}\mathrm{C}_{25} \mathrm{H}_{24} \mathrm{~N}_{8} \mathrm{O}_{4} \mathrm{~S} \\
532.58\end{array}$ & $\begin{array}{c}75 \\
285-7\end{array}$ & $\begin{array}{c}\text { C } 56.38 \\
\text { H } 4.54 \\
\text { N } 21.03\end{array}$ & $\begin{array}{c}56.19 \\
4.30 \\
20.99\end{array}$ & $\begin{array}{l}3423,3225\left(\mathrm{NH}_{2}, \mathrm{NHs}\right), \\
1696(\mathrm{CO}), 1440(\mathrm{~N}=\mathrm{N}- \\
\mathrm{N}), 1332,1143\left(\mathrm{SO}_{2}\right) .\end{array}$ \\
\hline $12 \mathrm{e}$ & & $\begin{array}{c}\mathrm{C}_{26} \mathrm{H}_{24} \mathrm{~N}_{6} \mathrm{O}_{5} \mathrm{~S} \\
532.57\end{array}$ & $\begin{array}{c}65 \\
237-9\end{array}$ & $\begin{array}{c}\text { C } 58.63 \\
\text { H } 4.54 \\
\text { N } 15.78 \\
\end{array}$ & $\begin{array}{c}58.51 \\
5.10 \\
15.33 \\
\end{array}$ & $\begin{array}{l}3422(\mathrm{NHs}), 1690(\mathrm{CO}), \\
1439(\mathrm{~N}=\mathrm{N}-\mathrm{N}), 1328, \\
1157\left(\mathrm{SO}_{2}\right) .\end{array}$ \\
\hline $15 \mathbf{a}$ & $\mathbf{H}$ & $\begin{array}{c}\mathrm{C}_{26} \mathrm{H}_{26} \mathrm{~N}_{6} \mathrm{O}_{5} \mathrm{~S} \\
534.58\end{array}$ & $\begin{array}{c}58 \\
280-2\end{array}$ & $\begin{array}{c}\text { C } 58.41 \\
\text { H } 4.90 \\
\text { N } 15.72\end{array}$ & $\begin{array}{c}\text { C } 58.69 \\
\text { H } 5.05 \\
\text { N } 15.62 \\
\end{array}$ & $\begin{array}{l}3500,3250\left(\mathrm{NH}_{2}, \mathrm{NHs}\right), \\
1688(\mathrm{CO}), 1439(\mathrm{~N}=\mathrm{N}- \\
\mathrm{N}), 1326,1146\left(\mathrm{SO}_{2}\right) .\end{array}$ \\
\hline $15 b$ & & $\begin{array}{c}\mathrm{C}_{30} \mathrm{H}_{28} \mathrm{~N}_{8} \mathrm{O}_{5} \mathrm{~S} \\
612.66\end{array}$ & $\begin{array}{c}71 \\
215-6\end{array}$ & $\begin{array}{l}\text { C } 58.81 \\
\text { H } 4.60 \\
\text { N } 18.28 \\
\end{array}$ & $\begin{array}{c}58.58 \\
4.91 \\
18.48 \\
\end{array}$ & $\begin{array}{l}3400,3224 \text { (NHs), } 1668 \\
(\mathrm{CO}), 1444(\mathrm{~N}=\mathrm{N}-\mathrm{N}), \\
1330,1142\left(\mathrm{SO}_{2}\right) .\end{array}$ \\
\hline $15 d$ & & $\begin{array}{c}\mathrm{C}_{32} \mathrm{H}_{32} \mathrm{~N}_{8} \mathrm{O}_{5} \mathrm{~S} \\
640.71\end{array}$ & $\begin{array}{c}66 \\
175-6\end{array}$ & $\begin{array}{l}\text { C } 59.98 \\
\text { H } 5.03 \\
\text { N } 17.48\end{array}$ & $\begin{array}{c}59.66 \\
4.79 \\
17.51\end{array}$ & $\begin{array}{l}3422(\mathrm{NHs}), 1654(\mathrm{CO}), \\
1436(\mathrm{~N}=\mathrm{N}-\mathrm{N}), 1326, \\
1150\left(\mathrm{SO}_{2}\right) .\end{array}$ \\
\hline
\end{tabular}


Table 1: Continued.

\begin{tabular}{|c|c|c|c|c|c|c|}
\hline \multirow{2}{*}{$\begin{array}{l}\text { Cpd. } \\
\text { No. }\end{array}$} & \multirow{2}{*}{$\mathrm{R}$} & \multirow{2}{*}{$\begin{array}{l}\text { Molecular } \\
\text { Formula } \\
\text { M. Wt. }\end{array}$} & \multirow{2}{*}{$\begin{array}{c}\text { Yield } \\
(\%) \\
\text { M.P. }\left({ }^{\circ} \mathrm{C}\right)\end{array}$} & \multicolumn{2}{|c|}{ Microanalysis (\%) } & \multirow{2}{*}{$\operatorname{IR}\left(\mathrm{cm}^{-1}\right)$} \\
\hline & & & & Calc. & Found & \\
\hline $15 d \mathbf{d}$ & & $\begin{array}{c}\mathrm{C}_{27} \mathrm{H}_{28} \mathrm{~N}_{8} \mathrm{O}_{5} \mathrm{~S} \\
576.63\end{array}$ & $\begin{array}{c}77 \\
255-6\end{array}$ & $\begin{array}{l}\text { C } 56.24 \\
\text { H } 4.89 \\
\text { N } 19.43 \\
\end{array}$ & $\begin{array}{c}56.20 \\
4.50 \\
19.42 \\
\end{array}$ & $\begin{array}{l}3424\left(\mathrm{NH}_{2}, \mathrm{NHs}\right), 1670 \\
(\mathrm{CO}), 1440(\mathrm{~N}=\mathrm{N}-\mathrm{N}), \\
1329,1141\left(\mathrm{SO}_{2}\right) .\end{array}$ \\
\hline $15 \mathrm{e}$ & & $\begin{array}{c}\mathrm{C}_{28} \mathrm{H}_{28} \mathrm{~N}_{6} \mathrm{O}_{6} \mathrm{~S} \\
576.17\end{array}$ & $\begin{array}{c}62 \\
195-7\end{array}$ & $\begin{array}{l}\text { C } 58.32 \\
\text { H } 4.89 \\
\text { N } 14.57\end{array}$ & $\begin{array}{l}\text { C } 58.59 \\
\text { H } 4.73 \\
\text { N } 14.57\end{array}$ & $\begin{array}{l}3421(\mathrm{NHs}), 1688(\mathrm{CO}), \\
1440(\mathrm{~N}=\mathrm{N}-\mathrm{N}), 1327, \\
1153\left(\mathrm{SO}_{2}\right) .\end{array}$ \\
\hline
\end{tabular}

Table 2. Physical, microanalytical and I.R. data of compounds 11a-h and 14a-j.

\begin{tabular}{|c|c|c|c|c|c|c|c|}
\hline \multirow{2}{*}{$\begin{array}{l}\text { Cpd. } \\
\text { No. }\end{array}$} & \multirow{2}{*}{$\mathrm{R}$} & \multirow{2}{*}{$\mathrm{R}^{\prime}$} & \multirow{2}{*}{$\begin{array}{l}\text { Molecular } \\
\text { Formula } \\
\text { M. Wt. }\end{array}$} & \multirow{2}{*}{$\begin{array}{l}\text { Yield } \\
(\%) \\
\text { M.P. } \\
\left({ }^{\circ} \mathrm{C}\right)\end{array}$} & \multicolumn{2}{|c|}{$\begin{array}{c}\text { Microanalysis } \\
(\%)\end{array}$} & \multirow{2}{*}{$\operatorname{IR}\left(\mathrm{cm}^{-1}\right)$} \\
\hline & & & & & Calc. & Found & \\
\hline 11a & & & $\begin{array}{c}\mathrm{C}_{33} \mathrm{H}_{32} \mathrm{~N}_{10} \mathrm{O}_{6} \mathrm{~S}_{2} \\
728.80\end{array}$ & $\begin{array}{c}235-6 \\
70\end{array}$ & $\begin{array}{l}\text { C } 54.38 \\
\text { H } 4.42 \\
\text { N } 19.21\end{array}$ & $\begin{array}{c}54.90 \\
4.25 \\
19.11\end{array}$ & $\begin{array}{l}3427,3335,3219 \mathrm{NH}_{2}, \\
\mathrm{NHs}), 1690(\mathrm{CO}), 1438 \\
(\mathrm{~N}=\mathrm{N}-\mathrm{N}), 1328,1138 \\
\left(\mathrm{SO}_{2}\right) .\end{array}$ \\
\hline $11 b$ & $\mathrm{H}$ & & $\begin{array}{c}\mathrm{C}_{36} \mathrm{H}_{34} \mathrm{~N}_{10} \mathrm{O}_{5} \mathrm{~S}_{2} \\
750.85\end{array}$ & $\begin{array}{c}65 \\
200-2\end{array}$ & $\begin{array}{l}\text { C } 57.58 \\
\text { H } 4.56 \\
\text { N } 18.65\end{array}$ & $\begin{array}{c}57.81 \\
4.45 \\
18.49 \\
\end{array}$ & $\begin{array}{l}3221,3074\left(\mathrm{NH}_{2}, \mathrm{NHs}\right), \\
1658(\mathrm{CO}), 1432(\mathrm{~N}=\mathrm{N}- \\
\mathrm{N}), 1314,1151\left(\mathrm{SO}_{2}\right) .\end{array}$ \\
\hline $11 \mathrm{c}$ & & & $\begin{array}{c}\mathrm{C}_{35} \mathrm{H}_{32} \mathrm{~N}_{12} \mathrm{O}_{5} \mathrm{~S}_{2} \\
764.84\end{array}$ & $\begin{array}{c}72 \\
215-7\end{array}$ & $\begin{array}{c}\text { C } 54.96 \\
\text { H } 4.21 \\
\text { N } 21.97 \\
\end{array}$ & $\begin{array}{c}54.81 \\
4.20 \\
21.90 \\
\end{array}$ & $\begin{array}{l}3216\left(\mathrm{NH}_{2}, \mathrm{NHs}\right), 1658 \\
(\mathrm{CO}), 1440(\mathrm{~N}=\mathrm{N}-\mathrm{N}), \\
1328,1151\left(\mathrm{SO}_{2}\right) .\end{array}$ \\
\hline 11d & & & $\begin{array}{c}\mathrm{C}_{37} \mathrm{H}_{36} \mathrm{~N}_{12} \mathrm{O}_{5} \mathrm{~S}_{2} \\
792.89\end{array}$ & $\begin{array}{c}71 \\
195-7\end{array}$ & $\begin{array}{l}\text { C } 56.04 \\
\text { H } 4.57 \\
\text { N } 21.19\end{array}$ & $\begin{array}{c}55.79 \\
4.59 \\
21.27\end{array}$ & $\begin{array}{l}3200\left(\mathrm{NH}_{2}, \mathrm{NHs}\right), 1678 \\
(\mathrm{CO}), 1440(\mathrm{~N}=\mathrm{N}-\mathrm{N}), \\
1326,1146\left(\mathrm{SO}_{2}\right) .\end{array}$ \\
\hline 11e & & $\mathrm{H}$ & $\begin{array}{c}\mathrm{C}_{31} \mathrm{H}_{30} \mathrm{~N}_{10} \mathrm{O}_{5} \mathrm{~S}_{2} \\
686.76\end{array}$ & $\begin{array}{c}75 \\
255-7\end{array}$ & $\begin{array}{c}\text { C } 54.21 \\
\text { H } 4.40 \\
\text { N } 20.39 \\
\end{array}$ & $\begin{array}{c}54.60 \\
4.70 \\
20.15 \\
\end{array}$ & $\begin{array}{l}3325,3218\left(\mathrm{NH}_{2}, \mathrm{NHs}\right), \\
1658(\mathrm{CO}), 1439(\mathrm{~N}=\mathrm{N}- \\
\mathrm{N}), 1316,1137\left(\mathrm{SO}_{2}\right) .\end{array}$ \\
\hline $11 f$ & & & $\begin{array}{c}\mathrm{C}_{37} \mathrm{H}_{36} \mathrm{~N}_{12} \mathrm{O}_{5} \mathrm{~S}_{2} \\
792.89\end{array}$ & $\begin{array}{c}74 \\
270-2\end{array}$ & $\begin{array}{l}\text { C } 56.04 \\
\text { H } 4.57 \\
\text { N } 21.19\end{array}$ & $\begin{array}{c}56.30 \\
4.88 \\
21.42 \\
\end{array}$ & $\begin{array}{l}3417,3327\left(\mathrm{NH}_{2}, \mathrm{NHs}\right), \\
1658(\mathrm{CO}), 1440(\mathrm{~N}=\mathrm{N}- \\
\mathrm{N}), 1326,1138\left(\mathrm{SO}_{2}\right) .\end{array}$ \\
\hline $11 \mathrm{~g}$ & & & $\begin{array}{c}\mathrm{C}_{34} \mathrm{H}_{32} \mathrm{~N}_{8} \mathrm{O}_{7} \mathrm{~S}_{2} \\
728.80\end{array}$ & $\begin{array}{c}69 \\
190-2\end{array}$ & $\begin{array}{l}\text { C } 56.03 \\
\text { H } 4.42 \\
\text { N } 15.37\end{array}$ & $\begin{array}{c}56.10 \\
4.62 \\
15.21\end{array}$ & $\begin{array}{l}3572,3276\left(\mathrm{NH}_{2}, \mathrm{NHs}\right), \\
1658(\mathrm{CO}), 1441(\mathrm{~N}=\mathrm{N}- \\
\mathrm{N}), 1329,1147\left(\mathrm{SO}_{2}\right) .\end{array}$ \\
\hline
\end{tabular}


Table 2: Continued.

\begin{tabular}{|c|c|c|c|c|c|c|c|}
\hline \multirow{2}{*}{$\begin{array}{l}\text { Cpd. } \\
\text { No. }\end{array}$} & \multirow{2}{*}{$\mathrm{R}$} & \multirow{2}{*}{$\mathrm{R}^{\prime}$} & \multirow{2}{*}{$\begin{array}{l}\text { Molecular } \\
\text { Formula } \\
\text { M. Wt. }\end{array}$} & \multirow{2}{*}{$\begin{array}{l}\text { Yield } \\
(\%) \\
\text { M.P. } \\
\left({ }^{\circ} \mathrm{C}\right) \\
\end{array}$} & \multicolumn{2}{|c|}{$\begin{array}{c}\text { Microanalysis } \\
(\%)\end{array}$} & \multirow{2}{*}{$\operatorname{IR}\left(\mathrm{cm}^{-1}\right)$} \\
\hline & & & & & Calc. & Found & \\
\hline $11 \mathrm{~h}$ & & $\mathrm{H}$ & $\begin{array}{c}\mathrm{C}_{34} \mathrm{H}_{30} \mathrm{~N}_{10} \mathrm{O}_{5} \mathrm{~S}_{2} \\
722.80\end{array}$ & $\begin{array}{c}72 \\
200-2\end{array}$ & $\begin{array}{l}\text { C } 56.49 \\
\text { H } 4.18 \\
\text { N19.37 }\end{array}$ & $\begin{array}{c}56.50 \\
4.00 \\
19.62\end{array}$ & $\begin{array}{l}3423,3280\left(\mathrm{NH}_{2}, \mathrm{NHs}\right), \\
1650(\mathrm{CO}), 1444(\mathrm{~N}=\mathrm{N}- \\
\mathrm{N}), 1334,1152\left(\mathrm{SO}_{2}\right) .\end{array}$ \\
\hline $14 a$ & & & $\begin{array}{c}\mathrm{C}_{34} \mathrm{H}_{36} \mathrm{~N}_{12} \mathrm{O}_{6} \mathrm{~S}_{2} \\
772.86\end{array}$ & $\begin{array}{c}66 \\
230-2\end{array}$ & $\begin{array}{l}\text { C } 52.83 \\
\text { H } 4.69 \\
\text { N21.74 }\end{array}$ & $\begin{array}{c}52.90 \\
4.70 \\
21.24\end{array}$ & $\begin{array}{l}3427,3337,3220\left(\mathrm{NH}_{2},\right. \\
\mathrm{NHs}), 1690(\mathrm{CO}), 1440 \\
(\mathrm{~N}=\mathrm{N}-\mathrm{N}), 1329,1138 \\
\left(\mathrm{SO}_{2}\right) .\end{array}$ \\
\hline $14 b$ & & & $\begin{array}{c}\mathrm{C}_{37} \mathrm{H}_{36} \mathrm{~N}_{12} \mathrm{O}_{6} \mathrm{~S}_{2} \\
808.89\end{array}$ & $\begin{array}{c}67 \\
245-7\end{array}$ & $\begin{array}{l}\text { C } 54.94 \\
\text { H } 4.48 \\
\text { N } 20.77\end{array}$ & $\begin{array}{c}54.83 \\
4.49 \\
20.47\end{array}$ & $\begin{array}{l}3428,3333,3219\left(\mathrm{NH}_{2},\right. \\
\mathrm{NHs}), 1658(\mathrm{CO}), 1433 \\
(\mathrm{~N}=\mathrm{N}-\mathrm{N}), 1332,1136 \\
\left(\mathrm{SO}_{2}\right) .\end{array}$ \\
\hline $14 c$ & & & $\begin{array}{c}\mathrm{C}_{35} \mathrm{H}_{36} \mathrm{~N}_{10} \mathrm{O}_{7} \mathrm{~S}_{2} \\
772.85\end{array}$ & $\begin{array}{c}66 \\
290-2\end{array}$ & $\begin{array}{c}\text { C } 54.39 \\
\text { H } 4.69 \\
\text { N } 18.12\end{array}$ & $\begin{array}{c}54.60 \\
4.50 \\
18.43\end{array}$ & $\begin{array}{l}3222\left(\mathrm{NH}_{2}, \mathrm{NHs}\right), 1678 \\
(\mathrm{CO}), 1440(\mathrm{~N}=\mathrm{N}-\mathrm{N}), \\
1330,1141\left(\mathrm{SO}_{2}\right)\end{array}$ \\
\hline 14d & & $\mathrm{H}$ & $\begin{array}{c}\mathrm{C}_{36} \mathrm{H}_{34} \mathrm{~N}_{10} \mathrm{O}_{6} \mathrm{~S}_{2} \\
766.21\end{array}$ & $\begin{array}{c}72 \\
160-2\end{array}$ & $\begin{array}{c}\text { C } 56.38 \\
\text { H } 4.46 \\
\text { N } 18.26 \\
\end{array}$ & $\begin{array}{c}56.75 \\
4.55 \\
18.26 \\
\end{array}$ & $\begin{array}{l}3190\left(\mathrm{NH}_{2}, \mathrm{NHs}\right), 1659 \\
(\mathrm{CO}), 1444(\mathrm{~N}=\mathrm{N}-\mathrm{N}), \\
1324,1178\left(\mathrm{SO}_{2}\right) .\end{array}$ \\
\hline $14 \mathrm{e}$ & & & $\begin{array}{c}\mathrm{C}_{40} \mathrm{H}_{36} \mathrm{~N}_{12} \mathrm{O}_{6} \mathrm{~S}_{2} \\
844.92\end{array}$ & $\begin{array}{c}75 \\
255-7\end{array}$ & $\begin{array}{l}\text { C } 56.86 \\
\text { H } 4.29 \\
\text { N } 19.89\end{array}$ & $\begin{array}{c}56.65 \\
4.55 \\
20.09\end{array}$ & $\begin{array}{l}3197\left(\mathrm{NH}_{2}, \mathrm{NHs}\right), 1678 \\
(\mathrm{CO}), 1441(\mathrm{~N}=\mathrm{N}-\mathrm{N}), \\
1326,1147\left(\mathrm{SO}_{2}\right) .\end{array}$ \\
\hline $14 f$ & $\mathrm{H}$ & & $\begin{array}{c}\mathrm{C}_{36} \mathrm{H}_{34} \mathrm{~N}_{10} \mathrm{O}_{6} \mathrm{~S}_{2} \\
766.21\end{array}$ & $\begin{array}{c}66 \\
200-2\end{array}$ & $\begin{array}{c}\text { C } 56.38 \\
\text { H } 4.46 \\
\text { N } 18.26 \\
\end{array}$ & $\begin{array}{c}56.13 \\
4.55 \\
18.26 \\
\end{array}$ & $\begin{array}{l}3383,3255\left(\mathrm{NH}_{2}, \mathrm{NHs}\right), \\
1653(\mathrm{CO}), 1435(\mathrm{~N}=\mathrm{N}- \\
\mathrm{N}), 1333,1156\left(\mathrm{SO}_{2}\right) .\end{array}$ \\
\hline $14 \mathrm{~g}$ & & & $\begin{array}{c}\mathrm{C}_{37} \mathrm{H}_{36} \mathrm{~N}_{12} \mathrm{O}_{6} \mathrm{~S}_{2} \\
808.89\end{array}$ & $\begin{array}{c}74 \\
230-2\end{array}$ & $\begin{array}{l}\text { C } 54.94 \\
\text { H } 4.48 \\
\text { N } 20.77 \\
\end{array}$ & $\begin{array}{c}54.60 \\
4.70 \\
20.87 \\
\end{array}$ & $\begin{array}{l}3250,3100\left(\mathrm{NH}_{2}, \mathrm{NHs}\right), \\
1688(\mathrm{CO}), 1444(\mathrm{~N}=\mathrm{N}- \\
\mathrm{N}) ; 1329,1151\left(\mathrm{SO}_{2}\right) .\end{array}$ \\
\hline $14 h$ & & & $\begin{array}{c}\mathrm{C}_{36} \mathrm{H}_{36} \mathrm{~N}_{8} \mathrm{O}_{8} \mathrm{~S}_{2} \\
772.85\end{array}$ & $\begin{array}{c}75 \\
255-7\end{array}$ & $\begin{array}{c}\text { C } 55.94 \\
\text { H } 4.69 \\
\text { N } 14.49 \\
\end{array}$ & $\begin{array}{c}55.60 \\
4.50 \\
14.12 \\
\end{array}$ & $\begin{array}{l}3263\left(\mathrm{NH}_{2}, \mathrm{NHs}\right), 1658 \\
(\mathrm{CO}), 1442(\mathrm{~N}=\mathrm{N}-\mathrm{N}), \\
1326,1154\left(\mathrm{SO}_{2}\right) .\end{array}$ \\
\hline $14 i$ & & & $\begin{array}{c}\mathrm{C}_{35} \mathrm{H}_{36} \mathrm{~N}_{10} \mathrm{O}_{7} \mathrm{~S}_{2} \\
772.85\end{array}$ & $\begin{array}{c}73 \\
267-9\end{array}$ & $\begin{array}{l}\text { C } 54.39 \\
\text { H } 4.69 \\
\text { N } 18.12 \\
\end{array}$ & $\begin{array}{c}54.58 \\
4.75 \\
18.21 \\
\end{array}$ & $\begin{array}{l}3329\left(\mathrm{NH}_{2}, \mathrm{NHs}\right), 1658 \\
(\mathrm{CO}), 1442(\mathrm{~N}=\mathrm{N}-\mathrm{N}), \\
1329,1154\left(\mathrm{SO}_{2}\right) .\end{array}$ \\
\hline $14 \mathbf{j}$ & & & $\begin{array}{c}\mathrm{C}_{39} \mathrm{H}_{40} \mathrm{~N}_{12} \mathrm{O}_{6} \mathrm{~S}_{2} \\
836.94\end{array}$ & $\begin{array}{c}73 \\
270-2\end{array}$ & $\begin{array}{l}\text { C } 55.96 \\
\text { H } 4.81 \\
\text { N } 20.08\end{array}$ & $\begin{array}{c}56.03 \\
5.05 \\
19.64\end{array}$ & $\begin{array}{l}3300,3200\left(\mathrm{NH}_{2}, \mathrm{NHs}\right), \\
1658(\mathrm{CO}), 1439(\mathrm{~N}=\mathrm{N}- \\
\mathrm{N}), 1327,1147\left(\mathrm{SO}_{2}\right) .\end{array}$ \\
\hline
\end{tabular}


assay is a sensitive measure of drug induced cytotoxicity with the best signal to noise ratio. The assay also, provides a colorimeteric end point that is nondestructive, indefinitely stable and visible to naked eye $\mathrm{e}^{25}$.

\section{Method}

Cells were plated in 97-muliwell plate $\left(10^{4}\right.$ cells/well $)$ for 24 hours before treatment with the compounds to allow attachment of cell to the wall of the plate. Different concentrations of the compound under test $(0,1,2.5$, 5 and $10 \mu \mathrm{g} / \mathrm{mL}$ ) were added to the cell monolayer. Triplicate wells were prepared for each individual dose. Monolayer cells were then incubated with the compound for 48 hours at $37^{\circ} \mathrm{C}$ and in atmosphere of $5 \% \mathrm{CO}_{2}$. After this time, cells were fixed, washed and stained with Sulforhodamie B stain. Excess stain was washed with acetic acid and attached stain was recovered with Tris EDTA buffer. The color intensity was measured in an LISA reader. Finally, the relation between surviving fraction and drug concentration was plotted to get the survival curve the tumor cell line after the specific compound.

\section{Analysis of data}

Data were collected, revised and analyzed by SPSS statistical package version 11. Excel computer program was used to tabulate the results (table 3), and represent it graphically (Figure 1-14). Probit regression analysis procedure has been introduced to select the best model that describes the relationship between the probit (percentages of protection) as a dependant variable in order to be used for prediction of the concentration of the drug that causes inhibition of $50 \%\left(\mathrm{IC}_{50}\right)$ or $90 \%$ $\left(\mathrm{IC}_{90}\right)$ of cancer cells. The in vitro growth inhibition properties of each drug were described by $\left(\mathrm{IC}_{50}\right)$ or $\left(\mathrm{IC}_{90}\right)$ and the degree of inhibition of cancer cell line was described by the equation:

The probit $(\mathrm{P})=$ intercept + (regression coefficient $\mathrm{x}$ conc).

\section{RESULTS AND DISCUSSION}

Data are presented in Table 3 to reveal the drug concentration and probit for the mammary cell line and illustrated by a series of curves (Fig. 1-Fig.14) as a plot of the probit against drug concentration of the tested compounds.

The data of the survival curves of the chosen compounds revealed that The $\mathrm{IC}_{50}$ compounds $\mathbf{3 a}, \mathbf{3 d}, \mathbf{4 a}, \mathbf{4 d}$, $5 \mathrm{a}$ and $5 \mathrm{~d}$ were 13.38, 14.41, 11.49, 12.73, 13.34 and 15.12 respectively. These results show that, all the tested triazeno-benzene sulfonamides are inactive except the unsubtituted sulfamoyl derivative $\mathbf{4 a}$ that possesses mild cytotoxic activity. In addition to, the monosubstituted piperazine derivative 4a has cytotoxic activity than its ethylcarboxylate analogue $\mathbf{3 a}$.

The 4-substituted carboxamide derivatives containing unsubstituted sulfamoyl derivatives 11a and 12a provide higher cytotoxic activity than those of the carbamimidoylsulfamoyl 
Table 3: Antitumor activity of the newly synthesized derivatives.

\begin{tabular}{||c|c|c|}
\hline Compound No & $\mathrm{IC}_{50}$ & $\mathrm{IC}_{90}$ \\
\hline $\mathbf{3 a}$ & 13.38 & 27.18 \\
\hline $\mathbf{3 d}$ & 14.41 & 28.81 \\
\hline $\mathbf{4 a}$ & 11.49 & 21.13 \\
\hline $\mathbf{4 d}$ & 12.73 & 22.75 \\
\hline $\mathbf{5 a}$ & 13.34 & 25.57 \\
\hline $\mathbf{5 d}$ & 15.12 & 30.71 \\
\hline $\mathbf{1 1 a}$ & 8.02 & 16.89 \\
\hline $\mathbf{1 1 b}$ & 8.61 & 20.32 \\
\hline $\mathbf{1 2 a}$ & 7.26 & 18.42 \\
\hline $\mathbf{1 2 d}$ & 9.89 & 20.46 \\
\hline $\mathbf{1 4 a}$ & 6.97 & 20.08 \\
\hline $\mathbf{1 4 f}$ & 15.42 & 29.69 \\
\hline $\mathbf{1 5 a}$ & 5.65 & 19.29 \\
\hline $\mathbf{1 5 d}$ & 7.91 & 20.02 \\
\hline
\end{tabular}

Fig.1-Fig.14. Cytotoxic activity of the newly synthesized derivatives.
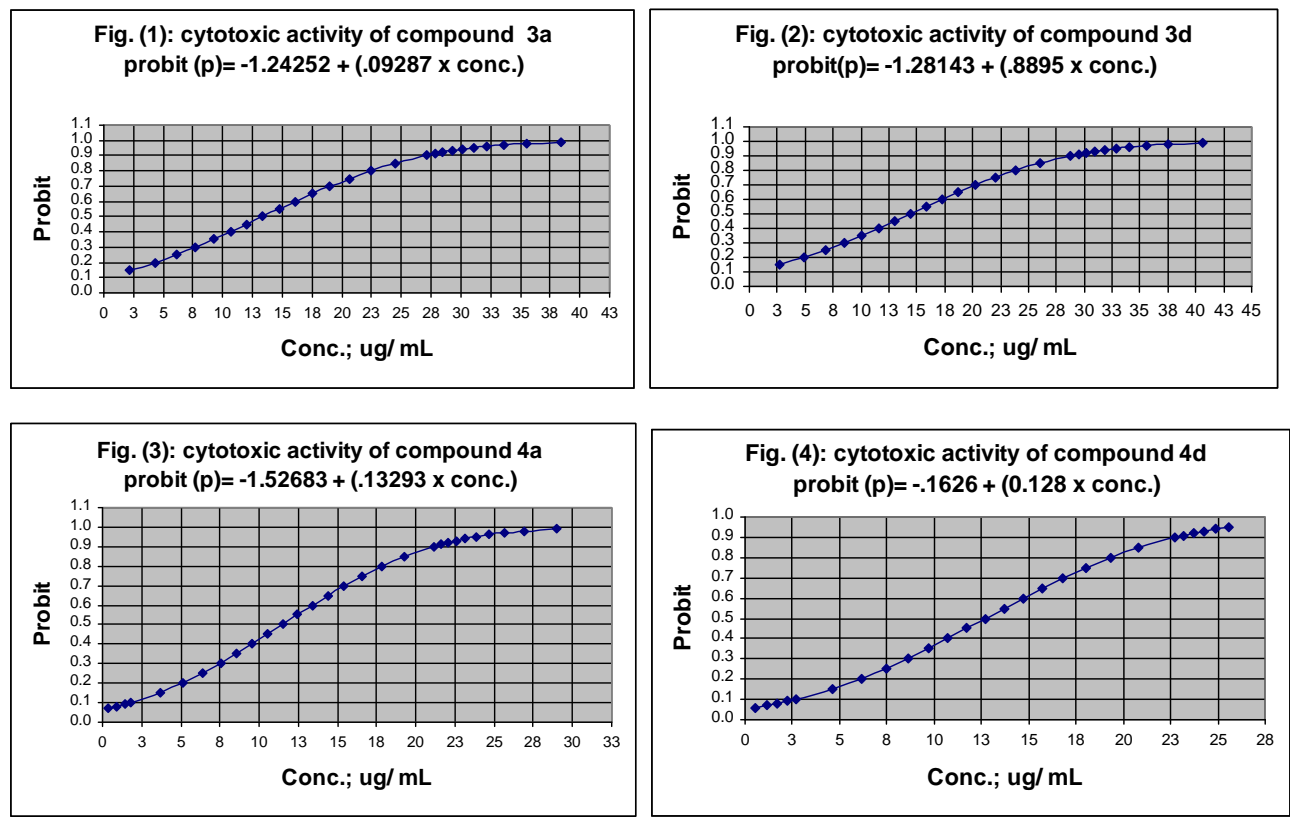

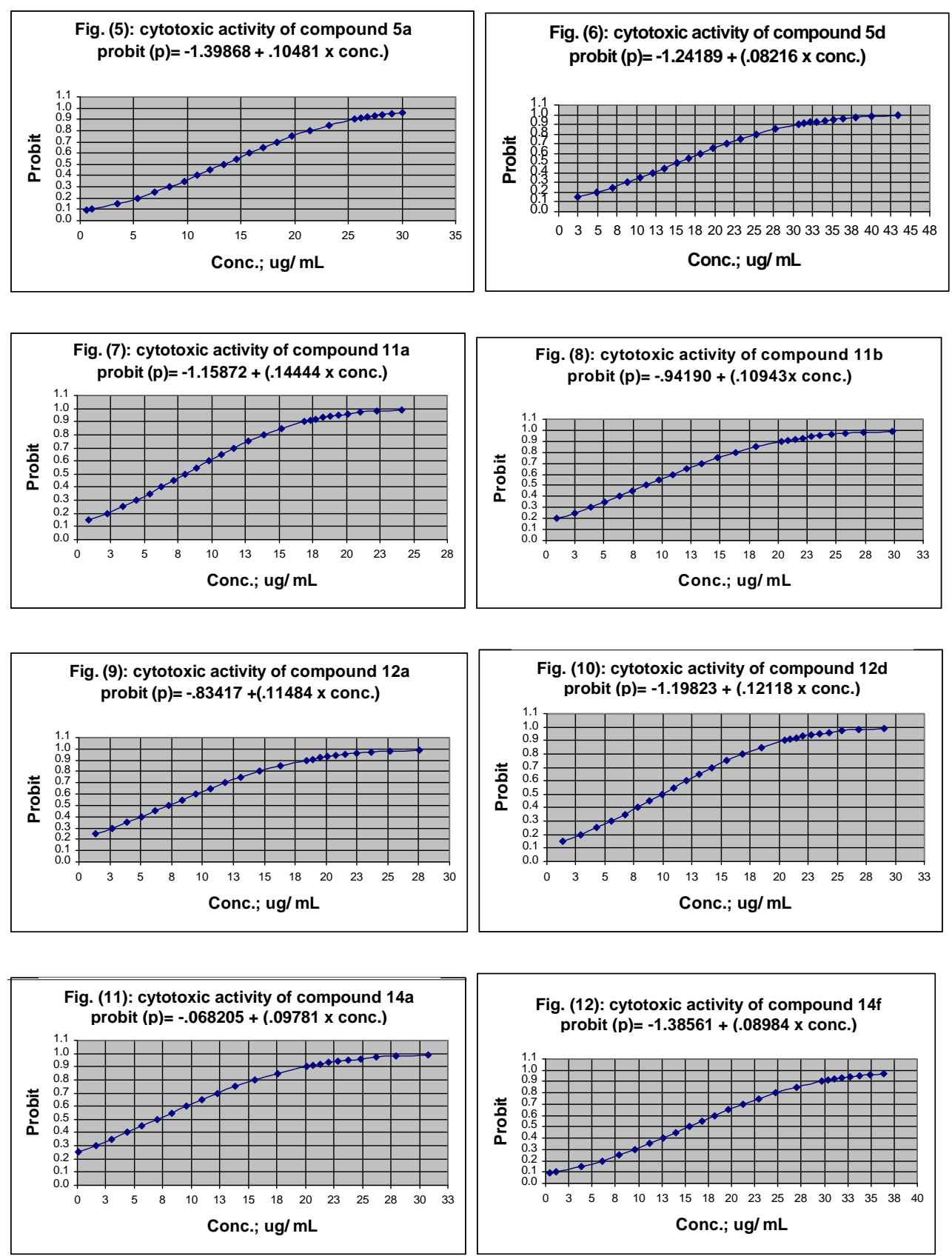


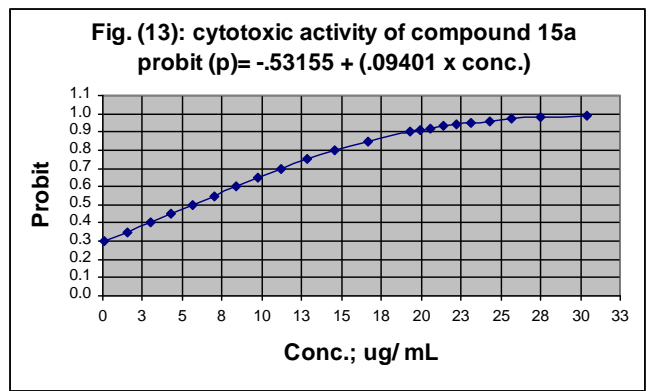

analogues $\mathbf{1 1 d}$ and $\mathbf{1 2 d}$ and $\mathrm{IC}_{50}$ for compounds 11a, 11b, 12a and 12d were $8.02,8.61,7.26$ and 9.89 respectively. These results indicate that, increasing the polarity of the compound decrease the cytotoxic activity. On the other hand, 11a shows a slight decrease in the cytotoxic activity than $\mathbf{1 1 b}$ attributed to the presence of 4,6-dimethyl-2pyrimidinyl group. Furthermore, $\mathrm{IC}_{50}$ for compounds 14a, 14f, 15a and 15d were $6.97,15.42,5.65$ and 7.91 respectively. These results show that similar to the 4-substituted carboxamide derivatives, the 4substituted ester derivatives containing unsubstituted sulfamoyl derivatives 15a shows higher cytotoxic activity than the carbamimidoylsulfamoyl analogue 15d. On the other hand, the 4substituted ester derivatives containing unsubstituted sulfamoyl derivatives 14f shows no cytotoxic activity compared to its carbamimidoylsulfamoyl analogue 14a that may be attributed to the presence of 2-pyrimidinyl moiety.

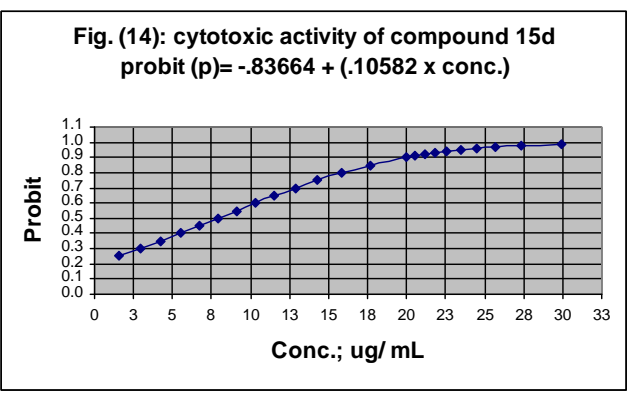

Finally, the 4-substituted ester derivative containing unsubstituted sulfamoyl derivatives 15a shows higher cytotoxic activity than its carboxamide analogue 14a which indicate that the lipophilicity increase the activity.

\section{Conclusion}

- The hybrid structure between an acridine nucleus and a triazene moiety results in an active cytotoxic combilexin, the case which complies with the aim of this work.

- Unsubstituted sulfamoyl derivatives show higher cytotoxic activity than the carbamimidoylsulfamoyl analogues.

- Subsitution in the 4 position of acridone by sulfamoylphenyl)diazenyl)piperazin-1-yl)ethyl group to form ester derivatives are more active than the 4-substituted sulfamoylphenyl)diazenyl)piperazi n-1-yl)carboxamide derivatives.

- Acridone derivatives are more potent than the substituted acridine derivatives.

- Lipophilicity increases the activity. 
- Finally it can be concluded that the cyotoxic activity decreases in the following order: 15a $>14 a>12 a$ $>15 d>11 b>11 a$ while two compounds show mild activity that are $\mathbf{1 2 d}>\mathbf{4 a}$.

\section{REFERENCES}

1- S. M. E. Moghazy Aly and H. M. Safwat, Egypt J. Pharm. Sci., 31, 505 (1990).

2- I. Antonini, P.Polucci, L.R. Kelland, S. Spinelli, N. Pescalli and S. Martelli, J. Med. Chem., 43, 4801 (2000).

3- J. B. Hansen, E. Langvad, F. Frandsen and O. Buchardt, ibid., 26, 1510 (1983).

4- G. J. Atwell, B. F. Cain, B. C. Baguley, G. J. Finlay and W. A. Denny, ibid., 27, 1481 (1984).

5- W. A. Denny, I. A. G. Roos and L. P. G. Wakelin; Anti-cancerDrug Res., 1, 141 (1986).

6- W. A. Denny, G. J. Atwell and B. C. Baguley, J. Med. Chem., 26, 1619 (1983).

7- G. J. Finlay, J. F. Rion, and B. C. Baguley, Eur. J. Cancer, Part A, 32, 708 (1996).

8- W.A. Denny, B. C. Baguley, B. F. Cain and R. J. Waring, "Molecular Aspects of Articancer Drug Action", Ed. Neidle and Waring, 1983, p. 1.

9- B. F. Cain, R. N. Seelye and G. J. Atwell, J. Med. Chem., 17, 922 (1974).
10- W. A. Denny, B. F. Cain, G. J. Atwell, C. Hansch, A. Panthananickal and A. Leo, ibid., 25, 276, (1982).

11- B. C. Baguley, W. A. Denny, G. J. Atwell, G. J. Finlay, G. W. Rewcastle, S. J. Twigden and W. R. Wilson, Cancer Res., 44, 3245 (1984).

12- E. Carvalho, A. P. Framcisco, J. Iley, E. Rosa, Bioorg. Med. Chem., 8, 1719 (2000).

13- V. Gadjeva and G. Vesselina, Int. J. Pharm., 247, 39 (2002).

14- P. Diana, P. Barraja, A. Lauria, A. M Almerico, G. Cirrinncione, A. Loi, C. Musiu, A. Pani, P. Lacolla and M. Marongiu, Eur. J. Med. Chem., 34, 353 (1999).

15- C. V. Catapano, M. Broggini, E. Erba, M. Ponti, L. Mariani, L. Citti and M. D'Incalci, Cancer Res., 47, 4884 (1987).

16- J. H. M. Van Delft, A. LuitenSchuite, V. L. Souliotis, S. A. Kyrtopoulos, J. Ouwerkerk, H. J. Keizer and R. A. Baan, Biomarkers, 1, 94, (1996)

17- V. R. Little and K. Vaughan, Can. J. Chem., 82, 1294 (2004).

18- I. Lalezari and F. Afghahi, J. Pharm. Sci., 64, 698 (1975).

19- A. W. McConnaughie and T. C. Jenkins, J. Med. Chem., 38, 3488 (1995).

20- G. J. Atwell, B. F. Cain and W. A. Denny, ibid., 20, 520, (1977).

21- K. Hano, A. Akashi, I. Yamamoto, S. Narumi, H. Iwata, Gann, 59, (3), 207 (1968), through Chem. Abst., 69, 425279 (1968). 
22- M. Y. Ebeid, S. M. El-Moghazy Aly, A. N. Mikhael and A. A. Eissa, Egypt. J. Pharm. Sci., 33, 293 (1992).

23- S. A Gamage, G. W. Reweastle, G. J. Atwell, B. C. Baguley and W. A. Denny, Anticancer Drug Des., 7, 403, (1992).
24- N. S. Drozdov and A. F. Bekhli; J. Gen. Chem. USSR, 8, 1505, (1938), through Chem. Abstr., 33, 45966 (1939).

25- H. Kalant and W. H. E. Roschlau, "Principles of Medical Pharmacology" $6^{\text {th }}$ edition, New York, Oxford, Oxford University Press, 1998, p. 774. 